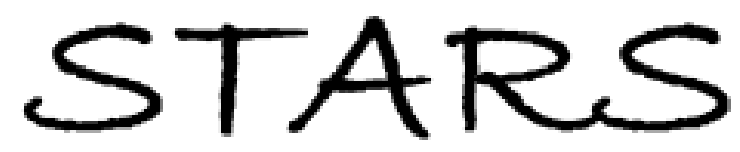

University of Central Florida

STARS

$1-1-1992$

\title{
Angle-Resolved Photoelectron Spectrometry Of Atomic Chlorine Using Synchrotron Radiation
}

P. Van der Meulen

M. O. Krause

C. D. Caldwell

University of Central Florida

S. B. Whitfield

University of Central Florida

C. A. de Lange

Find similar works at: https://stars.library.ucf.edu/facultybib1990

University of Central Florida Libraries http://library.ucf.edu

This Article is brought to you for free and open access by the Faculty Bibliography at STARS. It has been accepted for inclusion in Faculty Bibliography 1990s by an authorized administrator of STARS. For more information, please contactSTARS@ucf.edu.

\section{Recommended Citation}

Van der Meulen, P.; Krause, M. O.; Caldwell, C. D.; Whitfield, S. B.; and de Lange, C. A., "Angle-Resolved Photoelectron Spectrometry Of Atomic Chlorine Using Synchrotron Radiation" (1992). Faculty

Bibliography 1990s. 592.

https://stars.library.ucf.edu/facultybib1990/592

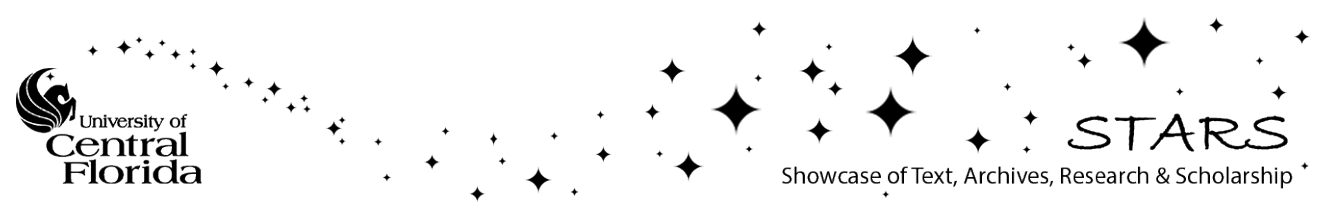




\title{
Angle-resolved photoelectron spectrometry of atomic chlorine using synchrotron radiation
}

\author{
P. van der Meulen \\ Chemistry Division, Oak Ridge National Laboratory, P.O. Box 2008, Oak Ridge, Tennessee 37831-6201 \\ and Department of Physical Chemistry, University of Amsterdam, Nieuwe Achtergracht 127, 1018 WS Amsterdam, The Netherlands \\ M. O. Krause \\ Chemistry Division, Oak Ridge National Laboratory, P. O. Box 2008, Oak Ridge, Tennessee 37831-6201 \\ C. D. Caldwell and S. B. Whitfield \\ Department of Physics, University of Central Florida, Orlando, Florida 32816 \\ C. A. de Lange \\ Department of Physical Chemistry, University of Amsterdam, Nieuwe Achtergracht 127, 1018 WS Amsterdam, The Netherlands
}

(Received 6 March 1992)

\begin{abstract}
Using synchrotron-radiation-based angle-resolved photoelectron spectrometry, the $\mathrm{Cl} 3 s 3 p^{5}\left({ }^{3} P_{2,1,0}^{o}\right) n p$ and $3 s 3 p^{5}\left({ }^{1} P_{1}^{o}\right) n p$ autoionizing Rydberg series are studied by monitoring the relative partial photoionization cross sections and asymmetry parameters of the $3 p^{-13} P_{2,1,0}^{e},{ }^{1} D_{2}^{e}$, and ${ }^{1} S_{0}^{e}$ ionic states between 21.75 and $27.75 \mathrm{eV}$. The Rydberg series converging upon the $3 s^{-1}{ }^{3} P_{2}^{o},{ }^{3} P_{1}^{o}$, and ${ }^{3} P_{0}^{o}$ thresholds are clearly resolved. Rydberg levels up to $n=14$ can be identified in the $\left({ }^{3} P_{2,1,0}^{o}\right) n p$ series, while the $\left({ }^{1} P_{1}^{o}\right) n p$ Rydberg series can be observed up to $n=10$. The relative partial cross sections are placed on an absolute scale using previous data obtained with an electron spectroscopy modulation method. The present experimental results for the $\mathrm{Cl}\left({ }^{3} P_{2,1,0}^{o}\right) n p$ and $\left({ }^{1} P_{1}^{o}\right) n p$ Rydberg series are compared with those of the Ar $3 s 3 p^{6}\left({ }^{2} S_{1 / 2}^{e}\right) n p$ series, and with theoretical results of a nonrelativistic many-body perturbation theory calculation. The ${ }^{3} P_{2,1,0}^{o}$ and ${ }^{1} P_{1}^{o}$ ionization thresholds obtained from the Rydberg series agree very well with the ionization limits calculated on the basis of the $\mathrm{Cl}$ II spectrum, and with the ionization energies determined from a $3 s^{-1}$ photoelectron spectrum. Autoionization phenomena due to $3 s^{2} 3 p^{5} \rightarrow 3 s^{2} 3 p^{3} n l n^{\prime} l^{\prime}$ two-electron excitations are observed in the ${ }^{1} P_{1}^{o}$ resonance region.
\end{abstract}

PACS number(s): $32.80 . \mathrm{Fb}, 32.80 . \mathrm{Dz}$

\section{INTRODUCTION}

The importance of electron correlations in the photoionization of closed-shell atoms is well established [1]. For open-shell systems their role has been demonstrated theoretically, but relatively few comparisons between theory and experiment have been made. In this perspective the photoionization of atomic chlorine is particularly interesting. Compared to the well-studied noble gas argon, atomic chlorine only lacks a single $3 p$ electron, which makes it an ideal model system for the incorporation of electron-correlation effects in the theoretical description of the photoionization of open-shell atoms. Hence, the photoionization of atomic chlorine has been investigated using a wide variety of theoretical methods [2-19], ranging from a straightforward independentparticle model [3] to a sophisticated many-body perturbation approach $[11,12]$.

However, due to the difficulties inherent in producing and handling such a reactive species, experimental determinations of partial photoionization cross sections $\left(\sigma_{i}\right)$ and asymmetry parameters $\left(\beta_{i}\right)$ of $\mathrm{Cl}$ are scarce or nonexistent. The first He I $\alpha(21.218 \mathrm{eV})$ photoelectron (PE) spectra of atomic chlorine were published in 1978 by De Leeuw, Mooyman, and De Lange [20] and by Kimura, Yamazaki, and Achiba [21]. The latter also presented relative differential cross sections for the $3 p^{-1}{ }^{3} P_{2,1,0}^{e}$ ${ }^{1} D_{2}^{e}$, and ${ }^{1} S_{0}^{e}$ ionic states measured at $90^{\circ}$ with respect to the unpolarized $\mathrm{He}$ I $\alpha$ photon beam. In an electron spectroscopy modulation experiment Van der Meer, Butselaar, and De Lange [22] determined the absolute photoionization cross section of the ${ }^{3} P_{2,1,0}^{e}$ components using $90^{\circ}$ data and calculated $\beta_{i}$ values. In 1988 Peel and Von Nagy-Felsobuki [23] published another He I $\alpha$ photoelectron spectrum of $\mathrm{Cl}$, but no absolute or relative cross sections were given. Only very recently [24] we have employed the continuum of synchrotron radiation to extend these photoelectron spectroscopic studies beyond the $\mathrm{He}$ I $\alpha$ energy. At present, no measurements of the $\beta_{i}$ parameters of the $3 p^{-1}$ ionic states have been reported. A more favorable situation exists with regard to the total photoionization cross section $(\sigma)$, which, using an experimentally less demanding mass-resolved ion detection technique, has been measured from threshold at $12.96 \mathrm{eV}$ to about $60 \mathrm{eV}[25,26]$.

Resonance structure due to autoionization phenomena provides direct evidence of electron correlations and is therefore of considerable interest. The energies of the autoionizing

$$
3 s^{2} 3 p^{4}\left({ }^{3} P_{2,1,0}^{e}, D_{2}^{e},{ }^{1} S_{0}^{3}\right) n s, n d
$$

Rydberg levels have been determined in photoabsorption 
[27-32] and photoionization [25] experiments. In addition to the resonance energies, the high-resolution ionyield measurements of Ruscic and Berkowitz [25] yielded information about the shapes and widths of these resonances. Theoretically, the

$$
3 s^{2} 3 p^{4}\left({ }^{3} P_{2,1,0}^{e},{ }^{1} D_{2}^{e},{ }^{1} S_{0}^{e}\right) n s, n d
$$

resonances have been studied in the $R$-matrix calculation of Lamoureux and Combet Farnoux [10]; the many-body perturbation theory (MBPT) calculation of Brown, Carter, and Kelly [12]; the eigenchannel quantum-defect analysis of Wang and Lu [14,15]; and the configurationinteraction (CI) calculation of Hansen et al. [18]. Satisfactory agreement between theory and experiment has been achieved [18], and the basic properties of the

$$
3 s^{2} 3 p^{4}\left({ }^{3} P_{2,1,0}^{e},{ }^{1} D_{2}^{e},{ }^{1} S_{0}^{e}\right) n s, n d
$$

Rydberg levels are well understood.

On the other hand, the autoionizing $3 s 3 p^{5}\left({ }^{3} P_{2,1,0}^{o},{ }^{1} P_{1}^{o}\right) n p(n \geq 4)$ Rydberg series were left virtually unstudied. For a long time the only experimental information related to these levels consisted of the location of the $3 s 3 p^{6}\left({ }^{2} S_{1 / 2}^{3)}\right.$ state [30] and the values of the $3 s^{-1}{ }^{3} P_{2,1,0}^{o}$ and ${ }^{1} P_{1}^{o}$ ionization energies [27,30,33], the ${ }^{1} P_{1}^{o}$ limit being the subject of some debate $[27,33]$. The only theoretical study addressing these resonances has been published by Brown, Carter, and Kelly [12]. Very recently [24] we reported the preliminary results of our electron spectrometry with synchrotron radiation (ESSR) studies of the $3 s \rightarrow n p$ autoionizing Rydberg series in $\mathrm{Cl}$. The $\left({ }^{3} P_{2,1,0}^{o}\right) n p$ resonances were studied by monitoring the ${ }^{3} P_{2,1,0}^{e}$ partial cross section between 21.75 and 24.75 eV. Good agreement was obtained with the theoretical level energies of Brown, Carter, and Kelly [12] and the ${ }^{3} P_{2,1,0}^{o}$ ionization energies derived from optical absorption experiments [27,30,33]. The $\left({ }^{3} P_{2,1,0}^{o}\right) n p$ Rydberg series in $\mathrm{Cl}$ was shown to resemble the analogous $3 s 3 p^{6}\left({ }^{2} S_{1 / 2}^{e}\right) n p$ series in Ar in several ways. However, resonances due to doubly excited states of the type $3 s^{2} 3 p^{3} n l n^{\prime} l^{\prime}$, which play a prominent role in the Ar spectrum around the $3 s^{-1}\left({ }^{2} S_{1 / 2}^{e}\right)$ ionization limit and were expected to be of importance in $\mathrm{Cl}$ as well, were not observed below the ${ }^{3} P_{2,1,0}^{o}$ thresholds.

In this paper we shall present a complete account of our study of the autoionization resonances just below the $3 s^{-1}{ }^{3} P_{2,1,0}^{o}$ and ${ }^{1} P_{1}^{o}$ ionization limits. These measurements are aimed at providing detailed information about the positions, shapes, and widths of the one- and twoelectron resonances of atomic chlorine in this energy region. It is hoped that a comparison between the present experiments and a sophisticated quantum-mechanical calculation [17] will enlarge our understanding of the role of electron correlations in the photoionization of openshell atoms.

The autoionization resonances are investigated by monitoring the partial cross sections of the $3 p^{-1}{ }^{3} P_{2,1,0}^{e}$, ${ }^{1} D_{2}^{e}$, and ${ }^{1} S_{0}^{e}$ ionic states between 21.75 and $27.75 \mathrm{eV}$. The partial cross sections are placed on an absolute scale using the results of the absolute $\mathrm{He} \mathrm{I} \alpha$ cross-section determination of Van der Meer, Butselaar, and De Lange
[22] modified by our experimental $\beta_{i}$ values. The $3 s 3 p^{5}\left({ }^{3} P_{2,1,0}^{o}\right) 5 p$ and $3 s 3 p^{5}\left({ }^{1} P_{1}^{o}\right) 4 p$ resonances are examined in detail, and the behavior of the asymmetry parameters across these resonances is obtained. Finally, we shall corroborate our analysis of the $\left({ }^{3} P_{2,1,0}^{o}\right) n p$ and the $\left({ }^{1} P_{1}^{o}\right)_{n p}$ Rydberg series by checking the ionization energies determined by fitting the level energies to the Rydberg formula against the ionization energies obtained from a Cl $3 s^{-1}$ photoelectron spectrum.

It should be noted that the photoionization of $\mathrm{Cl}$ atoms in the energy range of interest in the present experiments has also been studied by Samson, Shefer, and Angel [26] using a mass-spectrometry technique. However, because they employed a light source with a discrete spectrum, the resonances could not be observed. Furthermore, only the total cross section was obtained.

\section{EXPERIMENT}

Our electron spectrometry studies were performed at the Aladdin storage ring in Stoughton, Wisconsin. The experimental setup combines a microwave discharge tube [22] and an electron spectrometer [34], and has been described in some detail in our earlier work on atomic oxygen [35,36] and atomic bromine [37]. Briefly, the electron spectrometer contains three electrostatic analyzers mounted at right angles to each other on a rotatable platform which is perpendicular to the incoming photon beam. Within the dipole approximation the measured intensity $I_{i}(\theta)$ of the photoelectrons ejected from level $i$ at an angle $\theta$ with respect to the main polarization vector of the synchrotron radiation is then given by [38]

$$
I_{i}(\theta) \propto \frac{d \sigma_{i}}{d \Omega}=\frac{\sigma_{i}}{4 \pi}\left[1+\frac{\beta_{i}}{4}(1+3 p \cos 2 \theta)\right] .
$$

In this formula $d \sigma_{i} / d \Omega$ represents the differential cross section, $\sigma_{i}$ and $\beta_{i}$ are the (angle-integrated) partial photoionization cross section and asymmetry parameter of level $i$, and $p$ stands for the degree of linear polarization of the synchrotron radiation.

According to Eq. (1), the asymmetry parameter $\beta_{i}$ can be calculated from

$$
\beta_{i}=\frac{4\left(R_{i}-1\right)}{\left[3 p\left(R_{i}+1\right)-\left(R_{i}-1\right)\right]},
$$

where $R_{i}$ is the ratio of the intensities measured parallel and perpendicular to the electric-field vector. In our apparatus these intensities, i.e., $I_{i}\left(0^{\circ}\right)$ and $I_{i}\left(90^{\circ}\right)$, can be measured simultaneously. Using $\beta_{i}$ derived from Eq. (2), $\sigma_{i}$ can be obtained from the measurements at either $0^{\circ}$ or $90^{\circ}$ with the aid of Eq. (1). Alternatively, $\sigma_{i}$ can be measured directly at the so-called magic angle:

$$
\theta_{m}=\frac{1}{2} \cos ^{-1}\left(\frac{-1}{3 p}\right) \text {. }
$$

In general, the agreement between both schemes was excellent.

Chlorine atoms were produced in a $2.45-\mathrm{GHz} \mathrm{mi}-$ crowave discharge in a $\mathbf{C l}_{2}$ - $\mathrm{Ar}$ mixture. All gases were 
bought commercially and were used without further purification. The discharge was started with a spark from a Tesla coil and maintained in an 11-mm-diam quartz tube thinly coated with phosphorus pentoxide. The $\mathrm{Cl}$ atoms generated in the discharge were found to react rapidly with water molecules retained in the coating. Therefore, only a minimal amount of $\mathrm{P}_{2} \mathrm{O}_{5}$ was used. In addition, the coating was applied quickly to reduce the amount of moisture absorbed from the atmosphere. The microwave cavity was air cooled to prevent rapid deterioration of the coating. The dissipated microwave power amounted to about $70 \mathrm{~W}$. Argon was added to stabilize the discharge and enhance the atom yield. It also acted as a useful calibrator in the determination of the partial cross sections [39] and the asymmetry parameters [40].

The resulting gas mixture flowed into the ionization chamber through an 11-mm-diam Pyrex flow tube with a $5-\mathrm{mm}$ flow restriction at the low-pressure end to help maintain a pressure gradient between the discharge $\left(\approx 10^{-2}\right.$ Torr $)$ and the ionization region $\left(\approx 10^{-4}\right.$ Torr $)$. The flow tube of about $30 \mathrm{~cm}$ length was coated with halocarbon wax to minimize wall recombination reactions. The interior of the source cell, the slits, and the critical surfaces of the analyzers were coated with carbon to enhance spectrometer performance and help resist chemical attack. As determined from consecutive discharge-on and discharge-off spectra, about 40-50\% of the chlorine molecules entering the source cell were dissociated. The atom yield depended on the condition and the temperature of the $\mathrm{P}_{2} \mathrm{O}_{5}$ coating and the tuning of the microwave cavity. A schematic view of the experimental setup is shown in Fig. 1.

The PE spectra did not reveal evidence for excited atomic or excited molecular species. In particular, no signal due to the $\mathrm{Cl} 3 s^{2} 3 p^{5}\left({ }^{2} P_{1 / 2}^{o}\right)$ state, which lies 0.108 $\mathrm{eV}$ above the $3 s^{2} 3 p^{5}\left({ }^{2} P_{3 / 2}^{o}\right)$ ground state [27], was observed. It should be noted that at room temperature the equilibrium population of the ${ }^{2} P_{1 / 2}^{o}$ level is less than $1 \%$.

Because the dissociation of $\mathrm{Cl}_{2}$ molecules was incomplete, two sets of experiments were performed, namely, one with the microwave discharge on and another with the discharge off. Spectra virtually free of interfering signals due to undissociated $\mathrm{Cl}_{2}$ molecules could then be obtained by subtracting discharge-on and discharge-off spectra [41].

The resonances were studied using the constant-ionicstate (CIS) method [42] in which the photon energy and the photoelectron energy are advanced synchronously so as to stay on top of a particular peak in a photoelectron spectrum. This technique allows a fine-mesh scan of the autoionization features and has the advantage that the overall resolution is determined by the bandpass of the monochromator. Conventional photoelectron spectra were used for calibration and normalization purposes. Both CIS and PE spectra were taken in the constant pass energy mode, i.e., by scanning the (de)accelerating electric field the photoelectrons pass before entering the analyzers. The pass energy was typically $10.8 \mathrm{eV}$.

A monochromatic photon beam was provided by a 4-m normal-incidence monochromator (NIM) equipped with

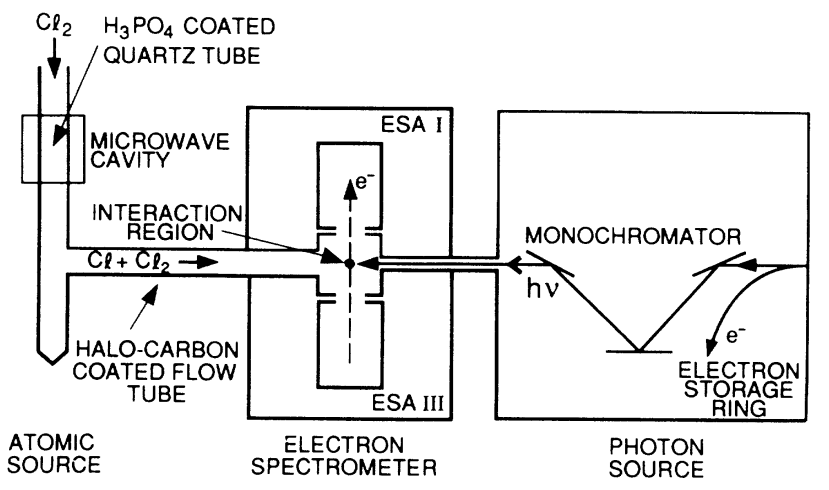

FIG. 1. Schematic view of the experimental setup.

a $3600-$ line $/ \mathrm{mm}$ gold-coated grating. The monochromator energy was calibrated against resonances in the spectra of Ar (Ref. 43) and Xe (Refs. 44 and 45) between 20 and $29 \mathrm{eV}$. The bandpass has been determined from the widths of the Ar $3 s 3 p^{6}\left({ }^{2} S_{1 / 2}^{e}\right) n p$ resonances and the $\mathrm{Xe}$ two-electron resonance at $20.665 \mathrm{eV}$ [44-48]. In the present experiments slit widths between 150 and $300 \mu \mathrm{m}$ were chosen, corresponding to a bandpass [full width at half maximum (FWHM)] between 0.09 and $0.16 \AA$. The monochromator slit function was well represented by a Gaussian.

The location of the $\theta=0^{\circ}$ axis [Eq. (1)] was established by measuring PE spectra as a function of the angle $\theta$ for the $\mathrm{Xe} 5 p_{3 / 2}$ photoline at $21.218 \mathrm{eV}$, which has a high $\beta_{i}$ value [49] and thus forms a sensitive indicator for the direction of the main polarization vector. The inhomogeneity of the source volume was determined in a similar experiment using the $\mathrm{Ne} 2 p$ photoline at $26.8 \mathrm{eV}$ for which $\beta_{i}$ is known to be zero [50,51]. Further, rare-gas calibration measurements at $21.218 \mathrm{eV}$ [49] served to obtain the degree of linear polarization of the synchrotron radiation and another correction factor related to the differences between the response functions of the analyzers. The measured polarization was $0.80 \pm 0.03$ at $21.218 \mathrm{eV}$. The calibrations were repeated several times during the experimental period.

In order to convert the measured magic-angle intensities $I_{i}\left(\theta_{m}\right)$ into relative partial photoionization cross sections, they had to be corrected according to

$$
\sigma_{i} \propto \frac{I_{i}\left(\theta_{m}\right)}{N(h v)},
$$

where $N(h v)$ is the relative number of photons at a particular energy. The function $N(h v)$ was determined using the "photoelectron spectrometry for the analysis of $\mathrm{x}$ rays" (PAX) method [52], in which the cross section of the Ar 3p level [39] was used. The relative cross sections were placed on an absolute scale using the results from previously performed electron spectroscopy modulation experiments [22].

\section{RESULTS AND DISCUSSION}

Figure 2 shows several typical $\theta=0^{\circ} \mathrm{PE}$ spectra taken at a photon energy of $21.75 \mathrm{eV}$. The upper panel (a) 
represents a spectrum of a $\mathrm{Cl}_{2}$ - $\mathrm{Ar}$ mixture recorded with the microwave discharge turned on. Strong features corresponding to the

$$
\mathrm{Cl} 3 s^{2} 3 p^{5}\left({ }^{2} P_{3 / 2}^{o}\right) \rightarrow \mathrm{Cl}^{+} 3 s^{2} 3 p^{4}\left({ }^{3} P_{2,1,0}^{e},{ }^{1} D_{2}^{e},{ }^{1} S_{0}^{3}\right)+e^{-}
$$

transitions at $E_{B} \approx 13.01,14.41$, and $16.42 \mathrm{eV}$ are easily identified $[20,21,23]$. In addition, signals associated with the photoionization of undissociated $\mathrm{Cl}_{2}$ molecules into the ${ }^{2} \Pi_{g, 3 / 2,1 / 2},{ }^{2} \Pi_{u, 3 / 2,1 / 2}$, and ${ }^{2} \Sigma_{g}^{+}$ionic states are observed at 11.6, 14.4, and $16.1 \mathrm{eV}$, respectively [53]. The $\mathrm{Cl}^{+}{ }^{1} D_{2}^{e}$ and ${ }^{1} S_{0}^{e}$ are seen to be partly obscured by the $\mathrm{Cl}_{2}^{+}{ }^{2} \Pi_{u, 3 / 2,1 / 2}$ and ${ }^{2} \Sigma_{g}^{+}$bands. A small $\mathrm{HCl}$ peak appears at about $12.8 \mathrm{eV}$. Presumably, $\mathrm{HCl}$ was formed mainly in a reaction between $\mathrm{Cl}$ atoms and moisture retained in the $\mathrm{P}_{2} \mathrm{O}_{5}$ wall coating. The $\mathrm{HCl}$ signal was found to decrease in the course of an experiment, most likely due to a gradual loss of water from the coating. The $\mathrm{HCl}$ bands partly overlap with the $\mathrm{Cl}$ atom peaks [21]. However, their intensity was very low so that only minor corrections were needed.

The middle panel (b) displays a pure $\mathrm{Cl}_{2}$ spectrum, while the lower panel (c) contains the spectrum obtained by subtracting (b) from (a). As can be seen from (c), all
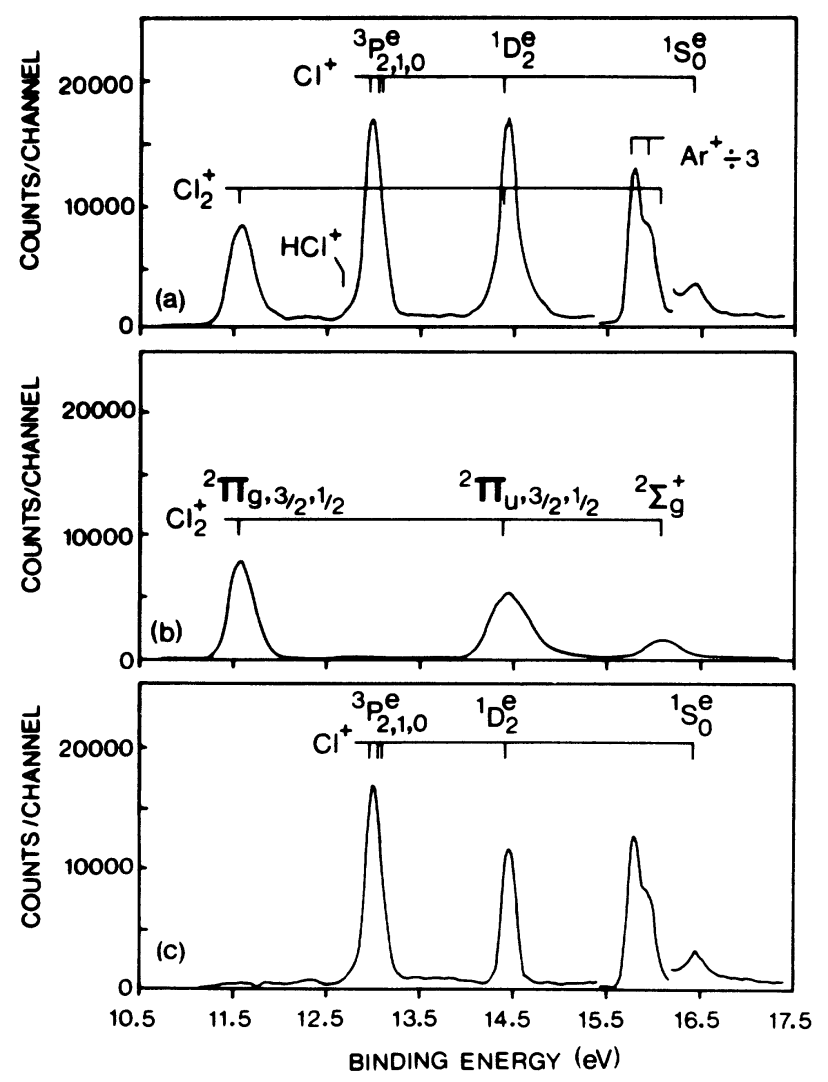

FIG. 2. Photoelectron spectra recorded at a photon energy of $21.75 \mathrm{eV}$ and at an angle of $0^{\circ}$ with respect to the main polarization vector of the synchrotron radiation: (a) microwave discharge on, (b) microwave discharge off, and (c) linear combination of discharge-on and discharge-off spectra in which the signals due to undissociated chlorine molecules have been removed. The spectra contain 256 channels each.
$\mathrm{Cl}_{2}$ bands are effectively removed from the combination spectrum.

Measured on the $\mathrm{Cl}^{+}\left({ }^{1} D_{2}^{e}\right)$ peak at $14.41 \mathrm{eV}$, the resolution (FWHM) of the combination spectrum is about $165 \mathrm{meV}$. This is approximately $30 \mathrm{meV}$ larger than the value expected on the basis of the normal analyzer resolution $(\Delta E / E=0.01$ ) and the broadening induced by the Doppler effect and the noisy environment of the storage ring, indicating that the spectrometer performance is negatively affected by the presence of the reactive $\mathrm{Cl}$ radicals. Nevertheless, reliable relative partial cross sections could be obtained by recording PE spectra at a relatively high pass energy of $10.8 \mathrm{eV}$, thereby shifting the spectra into the region of nearly constant analyzer transmission. Partly as a result of the high pass energy, the resolution is rather poor, and the $\mathrm{Cl}^{+} 3 p^{-1}{ }^{3} P_{2}^{e},{ }^{3} P_{1}^{e}$, and ${ }^{3} P_{0}^{e}$ peaks could not be separated. In the remainder of this paper they will be treated as a single photoline labeled ${ }^{3} P^{e}$.

\section{A. The photoionization parameters at $21.218 \mathrm{eV}$}

The relative partial photoionization cross sections and asymmetry parameters at the $\mathrm{He}$ I $\alpha$ energy $(21.218 \mathrm{eV})$ obtained in the present experiments are listed in Tables I and II, together with the results from previous experimental and theoretical studies. The uncertainty in our relative cross sections (normalized to 1.00 for the ${ }^{3} P^{e}$ ionic state) is about $6 \%$ for the ${ }^{1} D_{2}^{e}$ component and $20 \%$ for the ${ }^{1} S_{0}^{e}$ ionic state. The estimated probable error in the asymmetry parameters is about \pm 0.1 .

Our relative cross sections differ substantially from those obtained by Kimura, Yamazaki, and Achiba [21], and even after correction for angular distribution effects. Possible reasons for this discrepancy include the use by Kimura, Yamazaki, and Achiba [21] of the peak height, instead of the peak area, as a measure of the photoelectron intensity, and problems associated with the removal of the overlapping $\mathrm{Cl}_{2}^{+}\left({ }^{2} \Pi_{u, 3 / 2,1 / 2}\right)$ signal from the discharge-on spectrum of Kimura, Yamazaki, and Achiba [21] (see Fig. 2). It should be noted that in the case of atomic bromine [37], we also found an appreciably higher ${ }^{1} D_{2}^{e}$ cross section, although at that time the difference was attributed to the influence of nearby autoionizing resonances.

The present relative cross sections agree well with the open-shell transition-matrix (OSTM) calculation of Shahabi, Starace, and Chang [16] using experimental binding energies. On the other hand, most calculations seem to predict relative cross sections relatively close to the experimental values of Kimura, Yamazaki, and Achiba [21] and geometric ratios reflecting the degeneracies of the ionic states [54]. However, it is clear that there is little agreement among the calculations with regard to the relative cross sections. Both the ${ }^{1} D_{2}^{e}$ and ${ }^{1} S_{0}^{e}$ cross sections are seen to vary by more than a factor of 2 . In addition, many of the calculations show large differences between length and velocity results. Furthermore, calculations are often based on different, and sometimes unstated, values for the term energies. We also note that finalstate interchannel correlation effects, which have been shown to be very important in atomic chlorine [11,12], 
are not included in any Hartree-Fock (HF) -type calculation and in the random-phase approximation with exchange (RPAE) calculation of Starace and Armstrong [6].

Our experimental values for the asymmetry parameters are again in good agreement with the OSTM results of Shahabi and Starace [17], although $\beta\left({ }^{1} S_{0}^{e}\right)$ is overestimated by theory. This discrepancy for the ${ }^{1} S_{0}^{e}$ state may be attributed to its small cross section, which makes it particularly sensitive to the coupling with the strong ${ }^{3} P_{2,1,0}^{e}(\varepsilon s, \varepsilon d)$ and ${ }^{1} D_{2}^{3}(\varepsilon s, \varepsilon d)$ channels.

All calculations at the HF level $[3,13,17]$ as well as the RPAE calculation of Starace and Armstrong [6] correctly predict the asymmetry parameter for the ${ }^{3} P^{e}$ ionic state, but their results are clearly too high for the ${ }^{1} D_{2}^{e}$ and ${ }^{1} S_{0}^{e}$ components. The asymmetry parameters calculated by Fielder and Armstrong [13] using a multiconfiguration Hartree-Fock (MCHF) method are too low compared to the present experimental results. Thus, from our data, it appears that the most accurate description of the photoionization of atomic chlorine is obtained by Shahabi, Starace, and Chang [16] and by Shahabi and Starace [17] employing an OSTM formalism.

The relative cross sections were made absolute using $\sigma\left({ }^{3} P^{e}\right)$ at $21.218 \mathrm{eV}$ as determined by Van der Meer, Butselaar, and De Lange [22] in an electron spectroscopy modulation experiment based on the absolute cross section of $\mathrm{HCl}$ derived by Daviel et al. [55]. The absolute ${ }^{3} P^{e}$ cross section given by Van der Meer, Butselaar, and De Lange [22] was modified by using our present experimental value for the asymmetry parameter of the ${ }^{3} P^{e}$ ionic state, instead of the theoretical value of Fielder and Armstrong [13] employed in Ref. [22], to convert the measured differential cross section into a partial cross section. In this way we obtained $\sigma\left({ }^{3} P^{e}\right)=16.7 \mathrm{Mb}$. The absolute partial cross sections for the other ionic states were determined from the intensity ratios listed in Table I. Experimental and theoretical absolute photoionization

TABLE I. Experimental and theoretical (the geometric mean of length and velocity calculations is listed) partial cross-section ratios of atomic chlorine at $21.218 \mathrm{eV}$.

\begin{tabular}{|c|c|c|c|}
\hline Method & ${ }^{3} P_{2,1,0}^{e}$ & ${ }^{1} D_{2}^{e}$ & ${ }^{1} S_{0}^{e}$ \\
\hline Experiment $^{\mathrm{a}}$ & 1.00 & $0.69 \pm 0.04$ & $0.14 \pm 0.03$ \\
\hline Experiment $^{b}$ & 1.00 & 0.54 & 0.11 \\
\hline Experiment $^{\mathrm{c}}$ & 1.00 & 0.56 & 0.12 \\
\hline Geometric ratio & 1.00 & 0.56 & 0.11 \\
\hline Improved geometric ratio ${ }^{d}$ & 1.00 & 0.54 & 0.11 \\
\hline $\mathrm{HF}^{\mathrm{e}}$ & 1.00 & 0.37 & 0.10 \\
\hline RPAE $^{f}$ & 1.00 & 0.38 & 0.12 \\
\hline $\mathrm{HF}^{\mathrm{g}}$ & 1.00 & 0.53 & 0.15 \\
\hline $\mathrm{HF}(\text { relaxed })^{\mathrm{h}}$ & 1.00 & 0.51 & 0.12 \\
\hline HF ${\text { (unrelaxed })^{\mathrm{h}}}^{\mathrm{h}}$ & 1.00 & 0.34 & 0.08 \\
\hline MBPT $(\text { relaxed })^{\mathrm{i}}$ & 1.00 & 0.51 & 0.09 \\
\hline MBPT (unrelaxed) ${ }^{\mathrm{i}}$ & 1.00 & 0.47 & 0.08 \\
\hline $\mathrm{MCHF}^{\mathrm{j}}$ & 1.00 & 0.54 & 0.13 \\
\hline OSTM $^{\mathrm{k}}$ & 1.00 & 0.55 & 0.07 \\
\hline OSTM $^{1}$ & 1.00 & 0.70 & 0.12 \\
\hline Effective single-particle potential ${ }^{\mathrm{m}}$ & 1.00 & 0.59 & 0.12 \\
\hline Effective single-particle potential ${ }^{\mathrm{n}}$ & 1.00 & 0.60 & 0.10 \\
\hline
\end{tabular}

${ }^{\text {aT }}$ This work; estimated probable errors are given.

${ }^{b}$ Relative differential $\left(90^{\circ}\right)$ cross sections of Kimura, Yamazaki, and Achiba [21]; errors quoted as \pm 0.05 .

${ }^{c}$ Relative differential $\left(90^{\circ}\right)$ cross sections of Kimura, Yamazaki, and Achiba [21] corrected for angular distribution effects using the asymmetry parameters obtained in this work.

${ }^{\mathrm{d}}$ Improved geometric ratio of Berkowitz and Goodman [54].

${ }^{e}$ Hartree-Fock results from Starace and Armstrong [6].

${ }^{\mathrm{f}}$ Random-phase approximation with exchange results from Starace and Armstrong [6].

${ }^{\mathrm{g}} \mathrm{HF}$ results from Manson et al. [3].

${ }^{h} \mathrm{HF}$ results from Brown, Carter, and Kelly [12].

'Many-body perturbation theory results of Brown, Carter, and Kelly [12].

${ }^{\mathrm{j}}$ Multichannel Hartree-Fock results of Fielder and Armstrong [13].

${ }^{\mathrm{k}}$ Open-shell transition-matrix results of Shahabi, Starace, and Chang [16] using Hartree-Fock binding energies.

'OSTM results from Shahabi, Starace, and Chang [16] using experimental binding energies.

${ }^{\mathrm{m}}$ Effective single-particle potential results from Qian, Carter, and Kelly [19]; lowest-order calculation.

${ }^{n}$ Effective single-particle potential results from Qian, Carter, and Kelly [19]; lowest-order plus groundstate correlations. 
TABLE II. Experimental and theoretical (the geometric mean of length and velocity calculations is listed) asymmetry parameters of atomic chlorine at $21.218 \mathrm{eV}$.

\begin{tabular}{cccc}
\hline Method & ${ }^{3} P_{2,1,0}^{e}$ & ${ }^{1} D_{2}^{e}$ & ${ }^{1} S_{0}^{e}$ \\
\hline Experiment $^{\mathrm{a}}$ & 1.48 & 1.29 & 0.83 \\
& & & \\
HF $^{\mathrm{b}}$ & 1.43 & 1.73 & 1.74 \\
$\mathrm{RPAE}^{\mathrm{c}}$ & 1.49 & 1.77 & 1.76 \\
HF $^{\mathrm{d}}$ & 1.41 & 1.57 & 1.53 \\
HF $^{\mathrm{e}}$ & 1.41 & 1.65 & 1.72 \\
MCHF $^{\mathrm{f}}$ & 0.66 & 0.31 & 0.56 \\
HF $^{\mathrm{g}}$ & 1.43 & 1.70 & 1.69 \\
OSTM $^{\mathrm{h}}$ & 1.49 & 1.20 & 1.34 \\
\hline \hline
\end{tabular}

${ }^{a}$ This work; the estimated uncertainty in the experimental values is \pm 0.1 .

${ }^{b}$ Hartree-Fock results of Starace and Armstrong [6].

${ }^{c}$ Random-phase approximation with exchange results of Starace and Armstrong [6].

${ }^{\mathrm{d}} \mathrm{HF}$ results of Manson et al. [3].

${ }^{\mathrm{e}} \mathrm{HF}$ results of Fielder and Armstrong [13].

${ }^{f}$ Multichannel Hartree-Fock (MCHF) results of Fielder and Armstrong [13].

${ }^{\mathrm{g}} \mathrm{HF}$ results of Shahabi and Starace [17].

${ }^{h}$ Open-shell transition-matrix results of Shahabi and Starace [17].

cross sections of atomic chlorine are summarized in Table III.

Van der Meer, Butselaar, and De Lange [22] quoted an error of $13 \%$ in their absolute ${ }^{3} P^{e}$ cross section solely based on the statistical scatter in the

$$
\sigma\left(\mathrm{Cl}^{+},{ }^{3} \mathrm{P}^{e}\right) / \sigma\left(\mathrm{HCl}^{+},{ }^{2} \Pi_{3 / 2,1 / 2}\right)
$$

cross-section ratio and the uncertainty in the $\mathrm{HCl}$ standard [55]. Combining this value with the estimated probable error in $\beta\left({ }^{3} P^{e}\right)$ and the uncertainty in the relative cross sections, the absolute partial photoionization cross sections of the ${ }^{3} P^{e},{ }^{1} D_{2}^{e}$, and ${ }^{1} S_{0}^{e}$ ionic states are found to be accurate to $14 \%, 21 \%$, and $35 \%$, respectively.

The present experimental value for the asymmetry parameter of the ${ }^{3} P^{e}$ ionic state is considerably larger than the MCHF result of Fielder and Armstrong [13]. Consequently, our absolute ${ }^{3} P^{e}$ cross section is almost $18 \%$ smaller than the ${ }^{3} P^{e}$ cross section reported by Van der Meer, Butselaar, and De Lange [22] and, because possible errors introduced by the use of a theoretical $\beta\left({ }^{3} P^{e}\right)$ were not considered, even lies outside the error limits originally given in Ref. [22]. For the total photoionization cross section, obtained by adding the partial cross sections, the discrepancy with the results of Van der Meer, Butselaar, and De Lange [22] is reduced to about $8 \%$ due to an increase in the relative $\mathrm{Cl}^{+}{ }^{1} D_{2}^{e}$ cross section.

Compared to the absolute cross-section determination of Samson, Shefer, and Angel [26], our results for atomic chlorine are approximately $25 \%$ lower. Samson, Shefer, and Angel give an error of $8 \%$ for their averaged cross section, but the scatter in the experimental data points around $20 \mathrm{eV}$ is considerably larger. As will be shown below, these excursions are not caused by autoionization resonances. Although the error bars overlap slightly, the deviations between both absolute measurements [22,26] are substantial, and are at present not well understood. Van der Meer, Butselaar, and De Lange [22] used $\mathrm{HCl}$ (Ref. [55]) as a primary standard, while Samson, Shefer, and Angel [26] normalized the cross section of $\mathrm{Cl}$ atoms to one-half the molecular cross section [56]. It is therefore especially discomforting that the total absolute $\mathrm{Cl}_{2}$ cross section at $21.218 \mathrm{eV}$ obtained by Van der Meer, Butselaar, and De Lange [22] is about 25\% larger than the experimental value of Samson and Angel [56]. The situation in atomic chlorine is very similar to that in atomic oxygen, where the measurements of Van der Meer et al. [57] resulted in an absolute total photoionization cross section of oxygen atoms that was about $30 \%$ lower than the one determined by Samson and Pareek [58] and Angel and Samson [59]. Hence, there remains a considerable inconsistency among the various absolute cross sections measured for chalcogen and halogen atoms. On a relative scale, we can compare the total cross section given by Samson, Shefer, and Angel [26] with our $3 p^{-1}$ cross section obtained at 21.218 and at $21.75 \mathrm{eV}$, just above the ${ }^{1} P_{1}^{o}$ threshold. We observe a decrease in the cross section by a factor of $2.6(3)$, while a factor of 2.8 is obtained from the smoothed cross-section curve presented by Samson, Shefer, and Angel [26]. Actually, the agreement between both experiments is slightly worse than might be inferred from these numbers because the ion-yield measurements of Samson, Shefer, and Angel [26] also include the (small) contribution of the $\mathrm{Cl} 3 s$ subshell.

Nearly all calculations predict the total photoionization cross section of chlorine atoms to lie between 30 and $40 \mathrm{Mb}$. A clear trend, e.g., in going from calculations that do not include correlation effects to those that do, does not emerge. Most theoretical results are bracketed by the experimental values.

\section{B. The $3 s 3 p^{5}\left({ }^{3} P_{2,1,0}^{o}\right) n p$ resonance region}

Figure 3 shows the absolute partial cross sections of the $3 p^{-1}{ }^{3} P^{e},{ }^{1} D_{2}^{e}$, and ${ }^{1} S_{0}^{e}$ ionic states across the $3 s 3 p^{5}\left({ }^{3} P_{2,1,0}^{o}\right) n p(n \geq 4)$ autoionizing Rydberg series between 21.75 and $24.75 \mathrm{eV}$. These CIS spectra have been recorded at a resolution of $0.16 \AA$ (FWHM). The photon energy was advanced in steps of $5 \mathrm{meV}$. The estimated probable error in the relative cross sections at the initial and final points of the scan, as determined from PE spectra, is about $10 \%$ for each of the components. In a CIS scan the data are obtained by locking in upon the top of a particular peak in a PE spectrum, and because the ${ }^{3} P_{2,1,0}^{e}$ fine structure is not resolved (see Fig. 2), the ${ }^{3} P^{e}$ spectrum [Fig. 3(a)] corresponds to an ill-defined mean of the ${ }^{3} P_{2}^{e}$, ${ }^{3} P_{1}^{e}$, and ${ }^{3} P_{0}^{e}$ states. However, higher-resolution spectra recorded at a later date indicate that the signal observed is mostly due to the ${ }^{3} P_{2}^{e}$ component. The spectra shown are fully corrected and normalized. Specifically, molecular contributions could be reliably removed with the aid of discharge-off CIS spectra of the $\mathrm{Cl}_{2}^{+}{ }^{2} \Pi_{u, 3 / 2,1 / 2}$ and 
${ }^{2} \Sigma_{g}^{+}$bands recorded at the loci of the $\mathrm{Cl}^{+}{ }^{1} D_{2}^{e}$ and ${ }^{1} S_{0}^{e}$ ionic states. We note that the $\mathrm{Cl}_{2}^{+} \mathrm{CIS}$ spectra displayed several rather shallow and very broad window-type resonances in the 21.75-27.75-eV energy range which presumably covers excitations from the $\mathrm{Cl}_{2} 3 s \sigma_{u}$ band.

As can be seen in Fig. 3, the Rydberg series converging upon the ${ }^{3} \boldsymbol{P}_{2}^{o},{ }^{3} \boldsymbol{P}_{1}^{o}$, and ${ }^{3} \boldsymbol{P}_{0}^{o}$ thresholds are clearly resolved. The ${ }^{3} P_{2}^{o}$ series are well developed in all three channels, and Rydberg levels up to $n=14$ are identified. The series leading to the ${ }^{3} P_{1}^{o}$ limit are also easily distinguished, but only the first few members are observed. The ${ }^{3} P_{0}^{o}$ Rydberg series are rather weak and relatively broad. They can barely be detected in the ${ }^{3} P^{e}$ channel, but are visible in the ${ }^{1} D_{2}^{e}$ and ${ }^{1} S_{0}^{e}$ channels. Again, only the lower members can be identified. The pronounced resonance at $24.631 \mathrm{eV}$ corresponds to the first member of the Rydberg series converging upon the $3 s^{-1}{ }^{1} P_{1}^{o}$ threshold. This series will be discussed in more detail in Sec. III C.

The spin-orbit splitting in the ionic ${ }^{3} P_{2,1,0}^{o}$ core appears to be the dominant factor governing the overall appearance of the resonances for $n=5,6$, and 7 . Hence, the $3 s 3 p^{5}\left({ }^{3} P_{2,1,0}^{o}\right) n p$ levels should probably be discussed in term of a $J_{c} K$ coupling scheme [60-62]. In this coupling scheme the spin and orbital angular momenta of the ion core are first coupled to give the total angular momentum

TABLE III. Experimental and theoretical (the geometric mean of length and velocity calculations is listed) absolute photoionization cross sections (values given in $\mathrm{Mb}$ ) of atomic chlorine at $21.218 \mathrm{eV}$.

\begin{tabular}{|c|c|c|c|c|}
\hline Method & $\sigma\left({ }^{3} P_{2,1,0}^{e}\right)$ & $\sigma\left({ }^{1} D_{2}^{e}\right)$ & $\sigma\left({ }^{1} S_{0}^{e}\right)$ & $\sigma($ total $)$ \\
\hline Experiment $^{\mathrm{a}}$ & $16.7 \pm 2.5$ & $11.7 \pm 2.5$ & $2.4 \pm 0.8$ & $30.8 \pm 5.8$ \\
\hline Experiment ${ }^{\mathrm{b}}$ & $19.7 \pm 2.5$ & $11.4 \pm 1.5$ & $2.16 \pm 0.28$ & $33.3 \pm 4.2$ \\
\hline Experiment $\mathrm{t}^{\mathrm{c}}$ & & & & $38.5 \pm 3.1$ \\
\hline $\mathrm{CC}^{\mathrm{d}}$ & & & & 39 \\
\hline $\mathrm{HF}^{\mathrm{e}}$ & 22.6 & 8.3 & 2.3 & 33.2 \\
\hline RPAE $^{\mathrm{f}}$ & 20.6 & 7.8 & 2.5 & 30.9 \\
\hline RPAE $^{\mathrm{g}}$ & & & & 30 \\
\hline RPAE $^{h}$ & & & & 32.3 \\
\hline MBPT $^{\mathrm{i}}$ & & & & 37.8 \\
\hline $\mathrm{HF}^{\mathrm{j}}$ & 23.9 & 12.6 & 3.6 & 40.1 \\
\hline$R$-matrix ${ }^{\mathrm{k}}$ & & & & 38.7 \\
\hline$R$-matrix ${ }^{\prime}$ & & & & 35.0 \\
\hline $\mathrm{HF}(\text { relaxed })^{\mathrm{m}}$ & 17.7 & 9.1 & 2.1 & 28.9 \\
\hline $\mathrm{HF}\left(\right.$ unrelaxed) ${ }^{\mathrm{m}}$ & 18.4 & 6.3 & 1.5 & 26.2 \\
\hline MBPT $\left(\right.$ relaxed) ${ }^{n}$ & 20.1 & 10.2 & 1.8 & 32.1 \\
\hline MBPT $\left(\right.$ unrelaxed) ${ }^{\mathrm{n}}$ & 23.1 & 10.9 & 1.9 & 35.9 \\
\hline RPAE $^{\circ}$ & & & & 33 \\
\hline $\mathrm{MCHF}^{\mathrm{p}}$ & 20.4 & 10.9 & 2.6 & 33.9 \\
\hline $\mathrm{HF}^{\mathrm{q}}$ & & & & 26.8 \\
\hline $\mathrm{CC}^{\mathrm{r}}$ & & & & 35.8 \\
\hline OSTM $^{\mathrm{s}}$ & 19.7 & 13.8 & 2.4 & 35.9 \\
\hline Effective single-particle potential ${ }^{t}$ & 19.4 & 11.5 & 2.0 & 32.9 \\
\hline
\end{tabular}

${ }^{\mathrm{a}}$ This work; see text.

${ }^{\mathrm{b}}$ Experimental results of Van der Meer, Butselaar, and De Lange [22].

${ }^{\mathrm{c}}$ Experimental results of Samson, Shefer, and Angel [26].

${ }^{\mathrm{d}}$ Close-coupling results of Conneely, Smith, and Lipsky [5].

${ }^{\mathrm{e}}$ Hartree-Fock results of Starace and Armstrong [6].

${ }^{\mathrm{f}}$ Random-phase approximation with exchange results of Starace and Armstrong [6].

${ }^{g}$ RPAE results of Cherepkov and Chernysheva [7].

${ }^{h}$ RPAE results of Cherepkov and Chernysheva [8].

'Many-body perturbation theory results of Brown, Carter, and Kelly [11].

${ }^{\mathrm{j}} \mathrm{HF}$ results of Manson et al. [3].

${ }^{\mathrm{k}} R$-matrix results of Lamoureux and Combet Farnoux [10]; case I.

${ }^{1} R$-matrix results of Lamoureux and Combet Farnoux [10]; case II.

${ }^{\mathrm{m}} \mathrm{HF}$ results of Brown, Carter, and Kelly [12].

${ }^{n}$ MBPT results of Brown, Carter, and Kelly [12].

${ }^{\circ}$ RPAE results of Yarzhemsky and Nefedov [9].

${ }^{\mathrm{P}}$ Multichannel Hartree-Fock results of Fielder and Armstrong [13].

${ }^{q} \mathrm{HF}$ results of Shahabi, Starace, and Chang [16].

${ }^{\mathrm{r}} \mathrm{CC}$ results of Shahabi, Starace, and Chang [16].

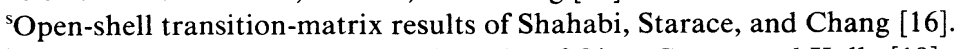

${ }^{t}$ Effective single-particle potential results of Qian, Carter, and Kelly [19]. 
$J_{c}$ of the core. Then the orbital angular momentum of the Rydberg electron is coupled to $J_{c}$ to give the resultant $K$. Finally, the spin of the Rydberg electron is coupled to $K$ to give the total angular momentum $J=K \pm \frac{1}{2}$. Generally, the splitting between the $K+\frac{1}{2}$ and the $K-\frac{1}{2}$ levels is quite small.

Physically, $J_{c} K$ coupling is appropriate if the spin-orbit interaction in the ionic core is much larger than the interaction between the core and the Rydberg electron. In particular, Rydberg levels with medium to high principal quantum numbers, when the Rydberg electron is, on the average, far removed from the core, are best classified according to their $J_{c} K$ quantum numbers [62]. In the specific case of the $\mathrm{Cl} 3 s \rightarrow n p$ Rydberg series, the excitation of the inner-valence $3 s$ electron enhances the effective nuclear charge experienced by the $3 p$ electrons and therefore causes a contraction of the $3 p$ subshell. This, in turn, leads to an increase of the interactions inside the core, and to a reduction of the interactions between the core and the Rydberg electron, thus making the $J_{c} K$ scheme applicable.

In case $J$ is a valid quantum number, only $n p$ Rydberg
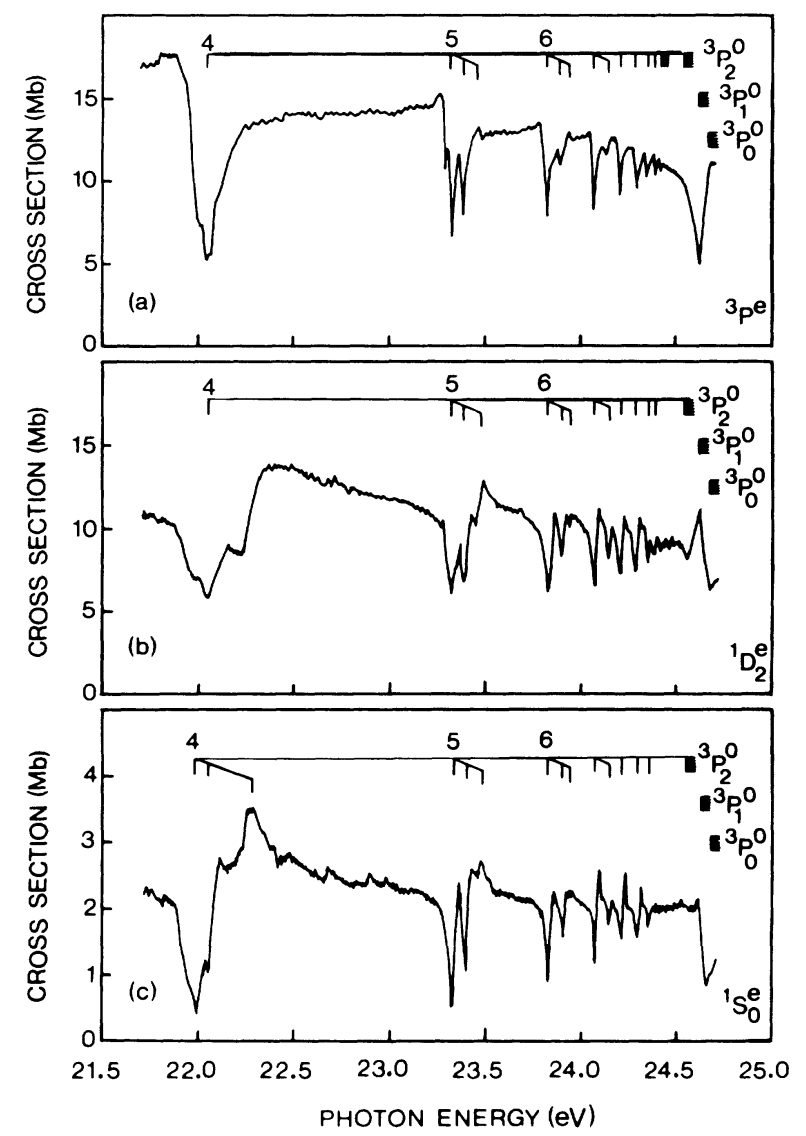

FIG. 3. Absolute partial cross sections of (a) the ${ }^{3} P^{e}$, (b) ${ }^{1} D_{2}^{e}$, and (c) ${ }^{1} S_{0}^{e}$ ionic states across the $3 s 3 p^{5}\left({ }^{3} P_{2,1,0}^{o}\right) n p(n \geq 4)$ autoionizing Rydberg series between 21.75 and $24.75 \mathrm{eV}$. The positions of the $\left({ }^{3} P_{2}^{o}\right) n p,\left({ }^{3} P_{1}^{o}\right) n p$, and $\left({ }^{3} P_{0}^{o}\right) n p$ Rydberg levels are indicated, as well as the locations of the $3 s^{-1}{ }^{3} P_{2}^{o},{ }^{3} P_{1}^{o}$, and ${ }^{3} P_{0}^{o}$ thresholds. These CIS spectra have been recorded at a resolution of $0.16 \AA$ (FWHM) and contain 600 channels each. states with $J=\frac{1}{2}, \frac{3}{2}$, or $\frac{5}{2}$ can be reached by one-photon absorption from the $\mathrm{Cl} 3 s^{2} 3 p^{5}\left({ }^{2} P_{3 / 2}^{o}\right)$ ground state. This brings the total number of accessible states of the $3 s 3 p^{5}\left({ }^{3} P_{2,1,0}^{o}\right) n p$ manifold up to 12 . Using $J_{c} K$ coupling, it can be shown that five states belong to each of the ${ }^{3} P_{2}^{o}$ and ${ }^{3} P_{1}^{o}$ cores, but that only two states have the ${ }^{3} P_{0}^{o}$ core as a parent.

Experimentally, we find a very broad $n=4$ resonance, indicative of many unresolved, overlapping levels. In comparison, the $n=5$ member displays a much more detailed structure and, although some additional features are partly resolved, the $3 s 3 p^{5}\left({ }^{3} P_{2}^{o}\right) 5 p, 3 s 3 p^{5}\left({ }^{3} P_{1}^{o}\right) 5 p$, and $3 s 3 p^{5}\left({ }^{3} P_{0}^{o}\right) 5 p$ levels are easily identified. The $6 p$ resonance is even more clearly resolved into three separate groups corresponding to the ${ }^{3} P_{2}^{o},{ }^{3} P_{1}^{o}$, and ${ }^{3} P_{0}^{o}$ core levels. Thus, it appears that the $J_{c} K$ scheme, which is a poor approximation for $n=4$, becomes more accurate for higher $n$.

For low $n(n \approx 4,5)$ the splitting between the states belonging to the same ion core is comparable to the energy separation between the core levels $(\approx 50-100 \mathrm{meV})$, leading to a very complex spectrum consisting of up to 12 lines for each $n p$ configuration and which should probably be described using an intermediate coupling scheme [60]. However, both this splitting and the width of the individual resonances (which is approximately inversely proportional to the third power of the effective quantum number $n^{*}$ ) decrease when the thresholds are approached, and for increasing $n(n \approx 5,6,7)$ the experimental spectrum is described progressively better by neglecting the fine structure and considering only three Rydberg "levels" for each $3 s 3 p^{5}\left({ }^{3} P_{2,1,0}^{o}\right) n p$ configuration, namely, $\left({ }^{3} P_{2}^{o}\right) n p,\left({ }^{3} P_{1}^{o}\right) n p$, and $\left({ }^{3} P_{0}^{o}\right) n p$. Nevertheless, it should always be remembered that these levels consist of five, five, and two nearly overlapping states, respectively. The $5 p$ resonance structure will be discussed in more detail below. For very large $n(n \geq 8)$ only the ${ }^{3} P_{2}^{o}$ series are observed. In this region the separation between the $\left({ }^{3} P_{2,1,0}^{o}\right) n p$ and the $\left({ }^{3} P_{2,1,0}^{o}\right)(n+1) p$ levels is comparable to the spin-orbit splitting of the core, which may give rise to further complications in the description of the spectrum.

Ignoring the fine structure, the experimental level energies, ionization energies, and the effective quantum numbers $n^{*}$ of the $3 s 3 p^{5}\left({ }^{3} P_{2,1,0}^{o}\right) n p$ Rydberg series are shown in Table IV. The level energies are a weighted average of the energies obtained for the individual ionic states, and may deviate slightly from the resonance energies reported earlier [24]. For resonances having a Lorentzian or window-type profile, the resonance energy is taken as the top or the bottom of the resonance, respectively. For dispersion-shape resonancs the resonance energy is assumed to be the energy at which the slope of the crosssection curve is maximal. The estimated error in the level energies is about $8 \mathrm{meV}$ for the ${ }^{3} P_{2}^{o}$ and ${ }^{3} P_{1}^{o}$ levels, but $20 \mathrm{meV}$ for the ${ }^{3} P_{0}^{o}$ states. These values include the contribution arising from the uncertainties in the resonance energies used for calibration $[44,45]$. The ionization energies and the effective quantum numbers are obtained by fitting the level energies $(n \geq 5)$ to the Rydberg formula, 
TABLE IV. Level energies $(E)$, ionization energies, and effective quantum number $\left(n^{*}\right)$ of the $\mathrm{Cl}$ $3 s 3 p^{5}\left({ }^{3} P_{2,1,0}^{o}\right) n p$ autoionizing Rydberg series.

\begin{tabular}{|c|c|c|c|c|c|c|c|c|}
\hline \multirow[b]{2}{*}{$n$} & \multicolumn{2}{|c|}{${ }^{3} P_{2}^{o}$ series $^{\mathrm{a}}$} & \multicolumn{2}{|c|}{${ }^{3} P_{1}^{o}$ series $^{\mathrm{a}}$} & \multicolumn{2}{|c|}{${ }^{3} P_{0}^{o}$ series ${ }^{a}$} & \multicolumn{2}{|c|}{${ }^{3} P^{o}$ series $^{b}$} \\
\hline & $E(\mathrm{eV})$ & $n^{*}$ & $E(\mathrm{eV})$ & $n^{*}$ & $E(\mathrm{eV})$ & $n^{*}$ & $E(\mathrm{eV})$ & $n^{*}$ \\
\hline 4 & 22.064 & 2.34 & & & & & 22.20 & 2.37 \\
\hline 5 & 23.339 & 3.35 & 23.400 & 3.32 & 23.490 & 3.38 & 23.45 & 3.39 \\
\hline 6 & 23.836 & 4.36 & 23.905 & 4.33 & 23.967 & 4.36 & 23.92 & 4.38 \\
\hline 7 & 24.080 & 5.37 & 24.154 & 5.34 & & & & \\
\hline 8 & 24.218 & 6.38 & & & & & & \\
\hline 9 & 24.300 & 7.35 & & & & & & \\
\hline 10 & 24.357 & 8.35 & & & & & & \\
\hline 11 & 24.398 & 9.4 & & & & & & \\
\hline 12 & 24.429 & 10.5 & & & & & & \\
\hline 13 & 24.449 & 11.5 & & & & & & \\
\hline 14 & 24.464 & 12.4 & & & & & & \\
\hline$\infty^{\mathrm{c}}$ & \multicolumn{2}{|c|}{$24.552 \pm 0.08$} & \multicolumn{2}{|c|}{$24.632 \pm 0.010$} & \multicolumn{2}{|c|}{$24.682 \pm 0.024$} & & \\
\hline$\infty^{d}$ & \multicolumn{2}{|c|}{24.544} & \multicolumn{2}{|c|}{24.622} & \multicolumn{2}{|c|}{24.663} & & \\
\hline
\end{tabular}

${ }^{a}$ This work; the estimated uncertainty in experimental level energies is about $\pm 8 \mathrm{meV}$ for the ${ }^{3} P_{2}^{o}$ and ${ }^{3} P_{1}^{o}$ Rydberg series, and $\pm 20 \mathrm{meV}$ for the ${ }^{3} P_{0}^{o}$ series.

${ }^{b}$ Theoretical results determined from Fig. 13 of Brown, Carter, and Kelly [12]; the experimental ionization energies used in this calculation and in the determination of $n^{*}$ are from Ref. [27]. The splitting of the ${ }^{3} P_{2,1,0}^{o}$ manifold is not considered in this nonrelativistic MBPT calculation.

${ }^{c}$ Ionization energies obtained by fitting the level energies to the Rydberg formula.

${ }^{\mathrm{d}}$ Optical spectroscopy values $[30,33]$ using a conversion factor of $1 \mathrm{eV}=8065.479 \mathrm{~cm}^{-1}$.

allowing the quantum defects $\delta=n-n^{*}$ to show a slight linear variation with photon energy [63]. For all three series, $\delta$ is equal to about 1.65 . This value is very close to the quantum defect of the analogous $3 s 3 p^{6}\left({ }^{2} S_{1 / 2}^{e}\right) n p$ Rydberg series in Ar [43], indicating that the $3 p$ subshell vacancy does not significantly alter the interaction between the core and the Rydberg electron.

Also included in Table IV are the experimental $3 s^{-1}$ ionization energies calculated on the basis of the $3 p^{-1}$ ${ }^{3} P_{2,1,0}^{e}$ ionization energies of $\mathrm{Cl}$ I [30] and the

$$
3 s^{2} 3 p^{4}\left({ }^{3} P_{2,1,0}^{e}\right) \rightarrow 3 s 3 p^{5}\left({ }^{3} P_{2,1,0}^{o}\right)
$$

excitation energies in $\mathrm{Cl}$ II [27,33], together with theoretical values presented by Brown, Carter, and Kelly [12] in Fig. 13 of Ref. [12]. The agreement with the optical spectroscopy values of Refs. [27], [30], and [33] is quite good. The nonrelativistic MBPT calculation of Brown, Carter, and Kelly [12] does not consider the spin-orbit splitting of the $3 s 3 p^{5}\left({ }^{3} P_{2,1,0}^{o}\right)$ core. In addition, the theoretical level energies may have to be shifted downwards by $50 \mathrm{meV}$ because the experimental $3 s^{-1}{ }^{3} P_{2,1,0}^{o}$ ionization energies used by Brown, Carter, and Kelly [12] were presumably based on the $3 p^{-1}{ }^{3} P_{2,1,0}^{e}$ ionization energies given by Moore [27] which have been superseded by the more accurate value of Radziemski and Kaufman [30]. Nevertheless, the calculated level energies agree well with those obtained in the present experiments.

From Fig. 3 it is clear that the strong ${ }^{3} P_{2}^{o}$ and ${ }^{3} P_{1}^{o}$ resonances are of the window type in all $3 p^{-1}{ }^{-1} P^{e},{ }^{1} D_{2}^{e}$, and ${ }^{1} S_{0}^{e}$ channels. The less pronounced ${ }^{3} P_{0}^{o}$ resonances seem to have a dispersion shape in the ${ }^{1} D_{2}^{e}$ channel, but a Lorentzian profile in the case of the ${ }^{1} S_{0}^{e}$ final state. They are also broader than the ${ }^{3} P_{2}^{o}$ and ${ }^{3} P_{1}^{o}$ resonances, making the level energies, and therefore the ${ }^{3} P_{0}^{o}$ ionization energy, less accurate.

The ${ }^{3} P_{2}^{o}$ and ${ }^{3} P_{1}^{o}$ resonances are best resolved in the ${ }^{1} S_{0}^{e}$ channel. In general, the $3 s 3 p^{5}\left({ }^{3} P_{2,1,0}^{o}\right) n p$ multiplet structure appears to be the simplest for the ${ }^{1} S_{0}^{e}$ ionic state, and most complex in the ${ }^{1} D_{2}^{e}$ channel. The ${ }^{1} S_{0}^{e}$ state is the only one for which the $\left({ }^{3} P_{2,1,0}^{o}\right) 4 p$ levels can be identified. For the other components the minimum of the broad $n=4$ resonance has been tentatively assigned to the $\left({ }^{3} P_{2}^{e}\right) 4 p$ level, while the $\left({ }^{3} P_{1,0}^{e}\right) 4 p$ states are not resolvable. The dissimilarities between the ionic states cannot easily be explained in terms of geometric arguments (angular momenta coupling, selection rules for autoionization, etc.) and clearly illustrate differences in the relevant matrix elements.

The absolute total photoionization cross section of atomic chlorine across the $3 s 3 p^{5}\left({ }^{3} P_{2,1,0}^{o}\right) n p(n \geq 4)$ resonances, obtained by adding the partial cross sections of Fig. 3, is portrayed in Fig. 4. The total cross section is dominated by the strong ${ }^{3} P_{2}^{o}$ and ${ }^{3} P_{1}^{o}$ window resonances. The ${ }^{3} P_{0}^{o}$ Rydberg series can hardly be detected.

The resonance profiles in the total cross section have been calculated by Brown, Carter, and Kelly [12]. Because they used $L S$ coupling, only three levels were considered for each $n p$ configuration, namely,

$$
3 s 3 p^{5}\left({ }^{3} P^{o}\right) n p\left({ }^{2} S^{e},{ }^{2} P^{e},{ }^{2} D^{e}\right) .
$$

Nevertheless, the line shape of the $n=4$ resonance is remarkably well reproduced, although experimentally the excursions are about twice as large. For the higher members, the spin-orbit splitting of the ${ }^{3} P_{2,1,0}^{o}$ core, which is omitted from the MBPT calculation [12], be- 


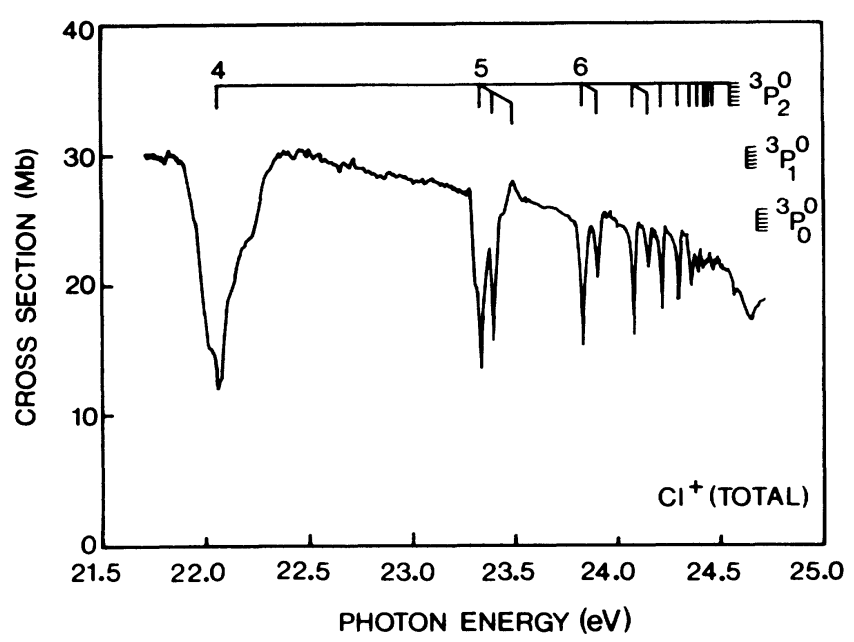

FIG. 4. Absolute total photoionization cross section of atomic chlorine across the $3 s 3 p^{5}\left({ }^{3} P_{2,1,0}^{o}\right) n p(n \geq 4)$ autoionizing Rydberg series between 21.75 and $24.75 \mathrm{eV}$. The total cross section has been obtained by adding the partial cross sections of Fig. 3 .

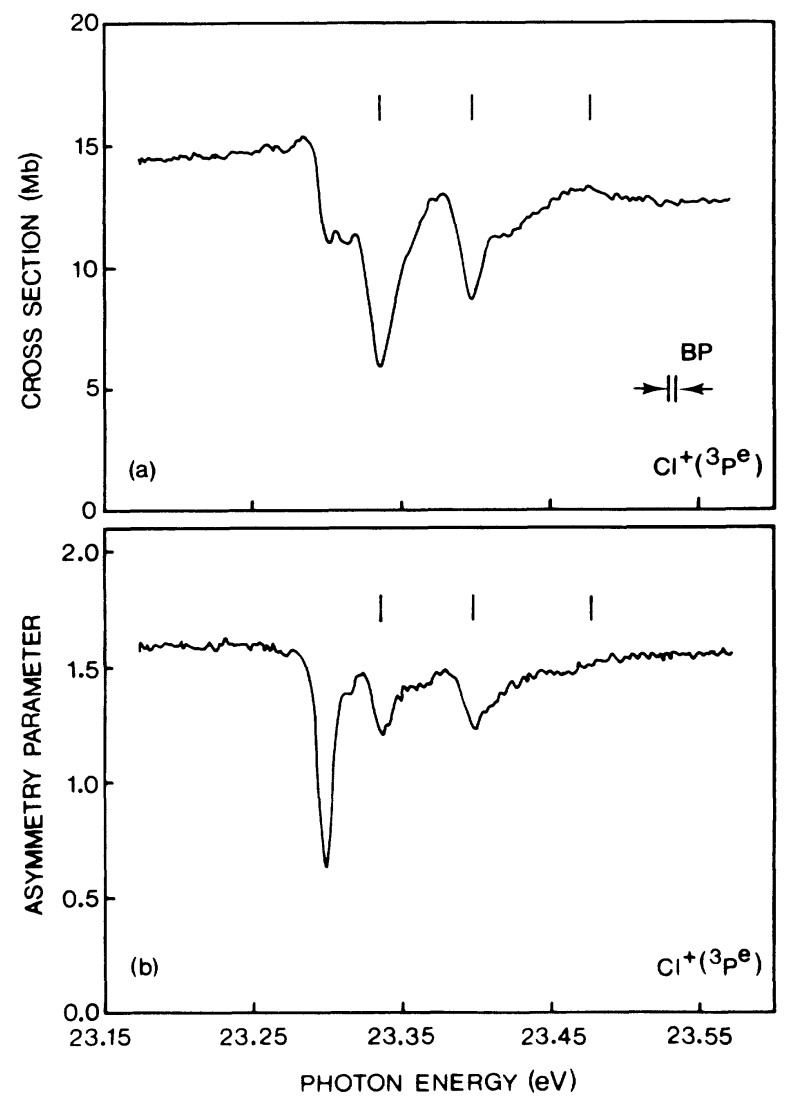

FIG. 5. (a) Absolute partial cross section and (b) asymmetry parameter of the $\mathrm{Cl}^{+}{ }^{3} \mathrm{P}^{e}$ ionic state across the $3 s 3 p^{5}\left({ }^{3} \mathrm{P}_{2,1,0}^{o}\right) 5 p$ autoionizing resonances between 23.20 and $23.60 \mathrm{eV}$. The estimated positions of the $\left({ }^{3} P_{2}^{o}\right) 5 p,\left({ }^{3} P_{1}^{o}\right) 5 p$, and $\left({ }^{3} P_{0}^{o}\right) 5 p$ resonances are indicated. BP represents the bandpass (BP) of $0.11 \AA$ (FWHM) at which these CIS spectra were recorded. The spectra contain 400 channels each. comes important and the agreement between the experimental and theoretical line shapes is less satisfactory.

Figures 5, 6, and 7 show the absolute photoionization cross sections and asymmetry parameters of the ${ }^{3} P^{e},{ }^{1} D_{2}^{e}$, and ${ }^{1} S_{0}^{e}$ ionic states across the $3 s 3 p^{5}\left({ }^{3} P_{2,1,0}^{o}\right) 5 p$ resonances. These spectra have been recorded at a resolution of $0.11 \AA$ (FWHM) and with a stepsize of $1 \mathrm{meV}$. The monochromator bandpass, which at these photon energies amounts to about $5.0 \mathrm{meV}$, is included in the figures. For most features the contribution of the monochromator is small, and the observed widths are almost equal to the natural widths. The smooth trace of the ${ }^{3} P^{e}$ spectrum provides a good example of the stability which, under favorable circumstances, can be achieved with our present discharge setup. The total photoionization cross section in the same region is displayed in Fig. 8.

The estimated positions of the $\left({ }^{3} P_{2}^{o}\right) 5 p,\left({ }^{3} P_{1}^{o}\right) 5 p$, and $\left({ }^{3} P_{0}^{o}\right) 5 p$ Rydberg levels are indicated in the figures. However, as can be readily seen in Figs. $5-8$, the $J_{c} K$ coupling scheme is only a zero-order approximation to the $3 s 3 p^{5}\left({ }^{3} P_{2,1,0}^{o}\right) 5 p$ manifold. The three groups of $5 p$ resonances are easily identified, but they are not fully separated, and fine structure is important. Hence, the $5 p$ positions obtained vary slightly among the ionic states de-
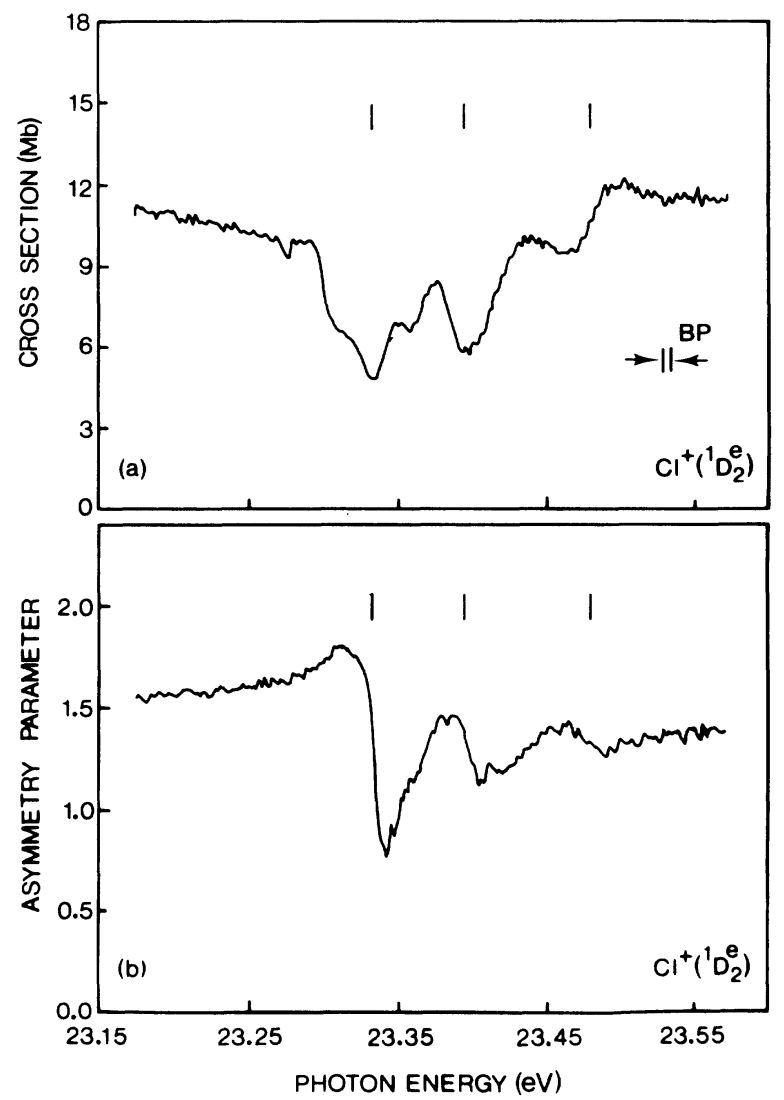

FIG. 6. (a) Absolute partial cross section and (b) asymmetry parameter of the $\mathrm{Cl}^{+}{ }^{1} D_{2}^{e}$ ionic state across the $3 s 3 p^{5}\left({ }^{3} P_{2,1,0}^{o}\right) 5 p$ autoionizing resonances between 23.20 and $23.60 \mathrm{eV}$. The estimated positions of the $\left({ }^{3} P_{2}^{o}\right) 5 p,\left({ }^{3} P_{1}^{o}\right) 5 p$, and $\left({ }^{3} P_{0}^{o}\right) 5 p$ resonances are indicated. BP represents the bandpass of $0.11 \AA$ (FWHM) at which these CIS spectra were recorded. The spectra contain 400 channels each. 
pending on the detailed resonance profiles. It seems that a proper description of the complex resonance structure will require an intermediate coupling scheme considering all 12 levels.

Most excursions in the asymmetry parameter of the ${ }^{3} P^{e}$ ionic state are not very strong. The most pronounced resonance belongs to an autoionizing state around 23.30 $\mathrm{eV}$, which can also be detected in the partial ${ }^{3} P^{e}$ cross section. The corresponding level of the $4 p$ manifold has been partially resolved as well (see Fig. 3), and together they form the first two members of a Rydberg series which presumably converges upon the ${ }^{3} P_{2}^{o}$ limit. This series is no longer resolved for $n \geq 5$, where it overlaps with the other series leading to the ${ }^{3} P_{2}^{o}$ threshold. The resonances in the asymmetry parameters of the ${ }^{1} D_{2}^{e}$ and ${ }^{1} S_{0}^{e}$ components generally coincide with the ${ }^{3} P_{2,1,0}^{o}$ level energies indicated in the figures.

The $5 p$ resonances in Figs. 5-8 have not been fitted [36,64] using Fano- [65-67] or Shore- [68-70] type expressions because the resonances due to autoionizing levels with the same total angular momentum $J$ are expected to interact strongly $[64,71]$. In other words, the overlap matrix defined by Mies [71] is nondiagonal. In general, the interaction between two autoionization resonances

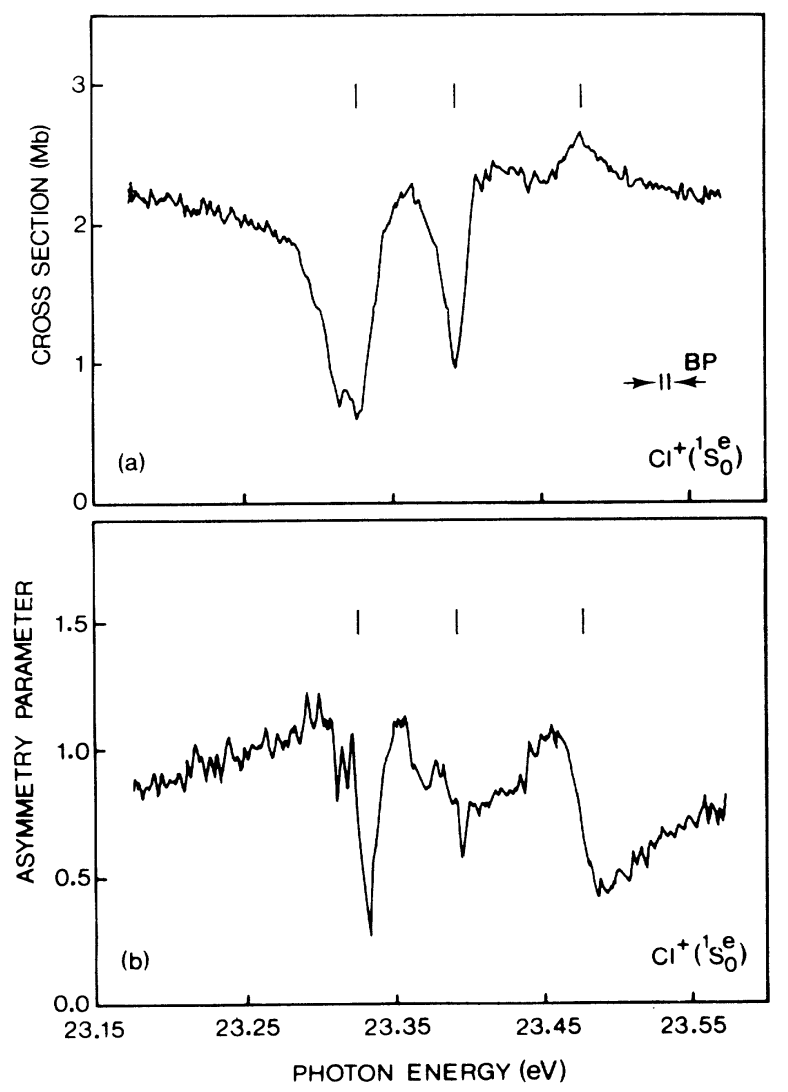

FIG. 7. (a) Absolute partial cross section and (b) asymmetry parameter of the $\mathrm{Cl}^{+}{ }^{1} S_{0}^{e}$ ionic state across the $3 s 3 p^{5}\left({ }^{3} P_{2,1,0}^{o}\right) 5 p$ autoionizing resonances between 23.20 and $23.60 \mathrm{eV}$. The estimated positions of the $\left.\left({ }^{3} P_{2}^{o}\right) 5 p,\left({ }^{3} P_{1}^{o}\right) 5 p\right)$ and $\left({ }^{3} P_{0}^{o}\right) 5 p$ resonances are indicated. BP represents the bandpass of $0.11 \AA$ (FWHM) at which these CIS spectra were recorded. The spectra contain 400 channels each.

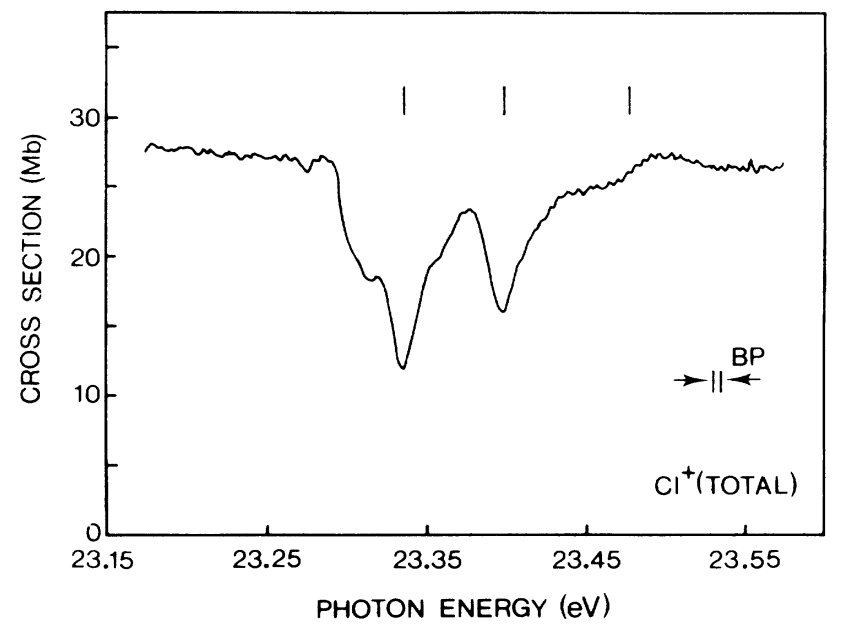

FIG. 8. Absolute total photoionization cross section of atomic chlorine across the $3 s 3 p^{5}\left({ }^{3} P_{2,1,0}^{o}\right) 5 p$ autoionizing resonances between 23.20 and $23.60 \mathrm{eV}$. The total cross section has been obtained by adding the partial cross sections of Figs. 5-7.

depends on their separation in relation to their true widths, and it is interesting to note that in going from $n=4$ to 7 , the interaction between resonances belonging to a different ionic core will decrease. Perhaps, for $n=6,7$, each group may even be treated as independent from all others. However, the interactions still present within the ${ }^{3} P_{2}^{o}$ and ${ }^{3} P_{1}^{o}$ groups preclude a straightforward parametrization of the resonance profiles. Because of symmetry considerations, both resonances belonging to the ${ }^{3} P_{0}^{o}$ group do not interact.

For comparison, the absolute partial photoionization cross section and the asymmetry parameter of the $\mathrm{Ar}^{+}$ $3 s^{2} 3 p^{5}\left({ }^{2} P_{3 / 2}^{o}\right)$ ionic state across the $3 s 3 p^{6}\left({ }^{2} S_{1 / 2}^{e}\right) 5 p$ resonances at $28.00 \mathrm{eV}$ are displayed in Fig. 9. These spectra have been recorded at a resolution of $0.09 \AA(5.5 \mathrm{meV})$ FWHM. Again, the bandpass of the monochromator is small compared to the natural width of the $5 p$ resonances [43]. The cross section has been made absolute using the absolute cross-section data of Marr and West [39] and our experimental $\sigma\left({ }^{2} P_{3 / 2}^{o}\right) / \sigma\left({ }^{2} P_{1 / 2}^{o}\right)$ cross-section ratio of $1.87 \pm 0.03$ at $28.78 \mathrm{eV}$. The behavior of the partial cross section and the asymmetry parameter of the ${ }^{2} P_{1 / 2}^{o}$ state across the $5 p$ resonances is nearly identical to that of the ${ }^{2} P_{3 / 2}^{o}$ component $[72,73]$. This means that, apart from an overall multiplication factor, Fig. 9(a) also represents the total photoionization cross section of $\mathrm{Ar}$ in the region of the $3 s 3 p^{6}\left({ }^{2} S_{1 / 2}^{e}\right) 5 p$ resonances. Note that Fig. 9 and Figs. 5-8 span the same energy intervals.

In the case of argon only two nearly overlapping states of the $3 s 3 p^{6}\left({ }^{2} S_{1 / 2}^{e}\right) 5 p$ manifold are accessible by onephoton absorption from the ${ }^{1} S_{0}^{e}$ ground state [43]. Hence, the spectrum is considerably simplified. Like the majority of the $5 p$ resonances in atomic chlorine, the $3 s 3 p^{6}\left({ }^{2} S_{1 / 2}^{e}\right) 5 p$ resonances in $\mathrm{Ar}$ are of window type. Taking into account that there are only two, physically merged, autoionizing levels, the $5 p$ resonances in $\mathrm{Ar}$ appear rather broad. In addition, the window in the Ar cross section is more pronounced.

To further compare the $5 p$ resonances in argon and 
chlorine, we have calculated the excess cross section [65-67] due to the interaction between the discrete $5 p$ levels and the available continua by integrating the total cross section across the complete $n=5$ manifold followed by subtracting the extrapolated contribution of the nonresonant cross section. Because the resonances are of window type, the excess cross section is negative. In this way, we obtained $-1.20 \mathrm{MbeV}$ for $\mathrm{Cl}$ and $-1.16 \mathrm{MbeV}$ for $\mathrm{Ar}$, corresponding to an oscillator strength of -0.0110 and -0.0105 . Obviously, these values depend on the normalization of the cross section and the nearperfect agreement may be fortuitous, but it seems that the $3 s 3 p^{5}\left({ }^{3} P_{2,1,0}^{o}\right) 5 p$ resonances in $\mathrm{Cl}$ and the $3 s 3 p^{6}\left({ }^{2} S_{1 / 2}^{e}\right) 5 p$ resonances in $\mathrm{Ar}$ are about equally strong. Interestingly, because of sum-rule arguments [74], the oscillator strength lost in the $n p$ resonances must reappear in some other part of the spectrum.

The $3 s 3 p^{5}\left({ }^{3} P_{2,1,0}^{o}\right) n p$ resonances could not be observed by Samson, Shefer, and Angel [26] and were not explicitly considered in their sum-rule analysis. However, because the total excess oscillator strength of the Rydberg series is unlikely to exceed -0.1 , this will not seriously affect their conclusions.

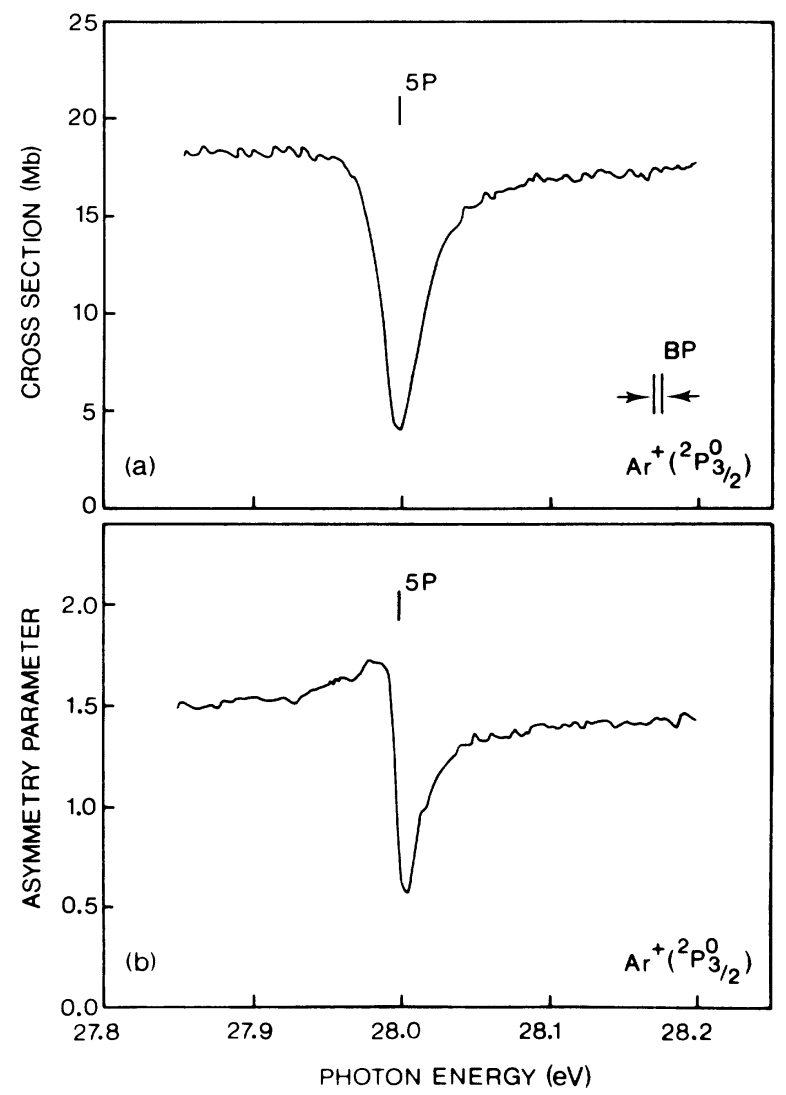

FIG. 9. (a) Absolute partial cross section and (b) asymmetry

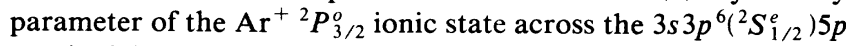
autoionizing resonances between 27.80 and $28.20 \mathrm{eV}$. The position of the minimum of the $5 p$ window at $27.998 \mathrm{eV}$ is indicated. BP represents the monochromator bandpass of $0.09 \AA$ (FWHM) at which these CIS spectra were recorded. The spectra contain 200 channels each.

\section{The $3 s 3 p^{5}\left({ }^{1} P_{1}^{o}\right) n p$ resonance region}

The absolute photoionization cross section of the $3 p^{-1}$ ${ }^{3} P^{e},{ }^{1} D_{2}^{e}$, and ${ }^{1} S_{0}^{e}$ ionic states between 24.25 and $27.75 \mathrm{eV}$ is shown in Fig. 10. These CIS spectra have been recorded at a resolution of $0.16 \AA$. The photon energy was advanced in steps of $5 \mathrm{meV}$. Apart from the clearly marked $3 s 3 p^{5}\left({ }^{1} P_{1}^{o}\right) n p(n \geq 4)$ autoionizing Rydberg series, many additional resonances are observed. In Fig. 10 their positions are indicated by vertical dashes, and they are labeled using the numbers 1-18. On the basis of energy considerations and in analogy with the spectrum of $\mathrm{Ar}$ (Ref. [43]), these resonances have been assigned to twoelectron excitations of the type

$$
3 s^{2} 3 p^{5}\left({ }^{2} P_{3 / 2}^{o}\right) \rightarrow 3 s^{2} 3 p^{3} n \ln ^{\prime} l^{\prime} \quad\left(J=\frac{1}{2}, \frac{3}{2}, \frac{5}{2}\right) .
$$

Using $J_{c} K$ coupling, it can be shown that there are five Rydberg series converging upon the ${ }^{1} P_{1}^{o}$ threshold. These Rydberg levels are not resolvable, although the very

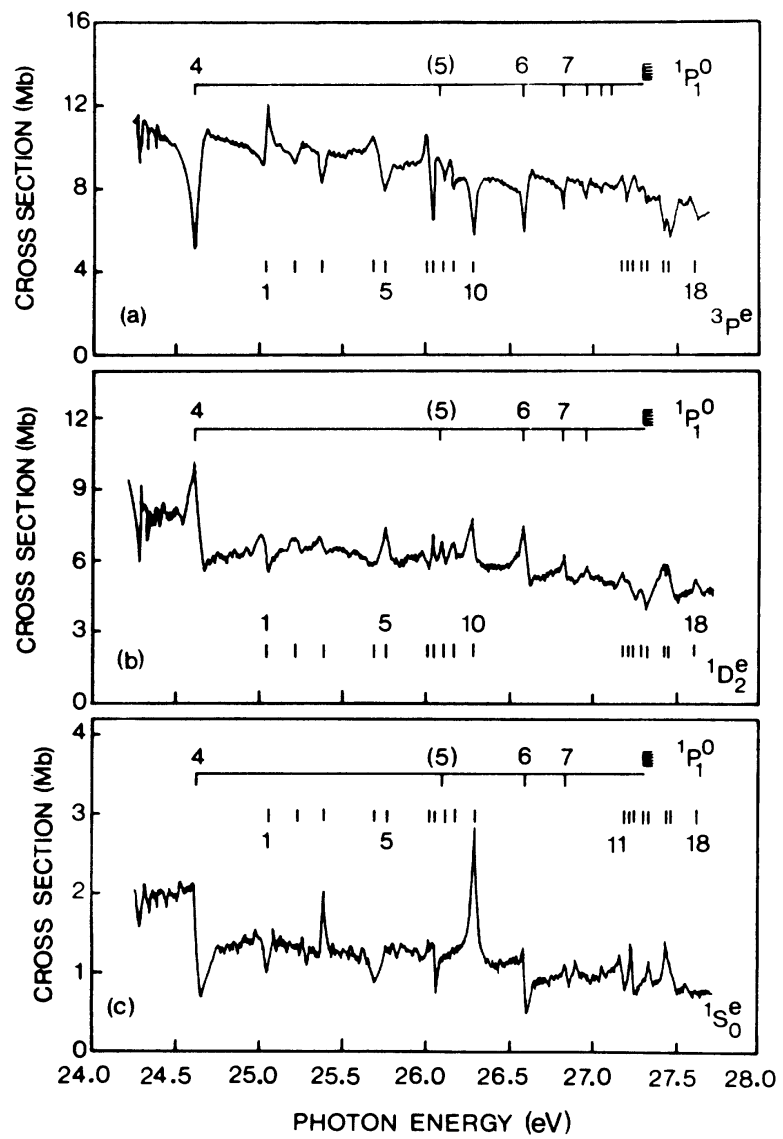

FIG. 10. Absolute partial cross sections of the (a) ${ }^{3} P^{e}$, (b) ${ }^{1} D_{2}^{e}$, and (c) ${ }^{1} S_{0}^{e}$ ionic states across the $3 s 3 p^{5}\left({ }^{1} P_{1}^{o}\right) n p(n \geq 4)$ resonance region between 24.25 and $27.75 \mathrm{eV}$. The positions of the $3 s 3 p^{5}\left({ }^{1} P_{1}^{o}\right) n p$ Rydberg levels, the $3 s^{2} 3 p^{3} n \ln ^{\prime} l^{\prime}$ doubly excited states, and the $3 s^{-1}{ }^{1} P_{1}^{o}$ ionization threshold are indicated. The $3 s 3 p^{5}\left({ }^{1} P_{1}^{o}\right) 5 p$ resonances could not be detected, but their calculated position is shown (see text). The two-electron resonances are labeled using the numbers 1-18. These CIS spectra have been recorded at a resolution of $0.16 \AA$ (FWHM) and contain 750 channels each. 
broad $n=4$ resonance exhibits some evidence of the more complex underlying structure, as will be shown below. The $3 s 3 p^{5}\left({ }^{1} P_{1}^{o}\right) n p$ Rydberg series is best developed in the ${ }^{3} P^{e}$ channel in which resonances up to $n=10$ can be identified. For the ${ }^{1} D_{2}^{e}$ and ${ }^{1} S_{0}^{e}$ ionic states the $n p$ Rydberg series extend to, respectively, $n=8$ and 7. However, the $n=5$ resonance cannot be detected, presumably due to interactions with nearby doubly excited states. The estimated position of the $5 p$ resonances, based on the ${ }^{1} P_{1}^{o}$ limit and the quantum defect of the $\left({ }^{1} P_{1}^{o}\right) n p$ Rydberg series (see below), is indicated in Fig. 10. Several resonances have the approximately correct energy, but none of them matches the profile of the other members of the Rydberg series in all three channels. Hence, they are attributed to doubly excited $3 s^{2} 3 p^{3} n l n^{\prime} l^{\prime}$ states. Rydberg levels with $n \geq 6$ appear to lie in a relatively clean part of the spectrum and should be unperturbed. It should be noted that Rydberg series with one or two missing members were also observed in $\mathrm{Ar}$ [43].

The one-electron $3 s 3 p^{5}\left({ }^{1} P_{1}^{o}\right) n p$ resonances have a window-type profile in the ${ }^{3} P^{e}$ channel, whereas they display a dispersion shape in the ${ }^{1} D_{2}^{e}$ and ${ }^{1} S_{0}^{e}$ channels. This should be compared to the $3 s 3 p^{5}\left({ }^{3} P_{2,1,0}^{o}\right) n p$ resonances which, as far as the strong ${ }^{3} P_{2}^{o}$ and ${ }^{3} P_{1}^{o}$ resonances are concerned, have a similar shape in all channels.

The $3 s 3 p^{5}\left({ }^{1} P_{1}^{o}\right) 4 p$ resonances are located in the center of the $3 s^{-1}{ }^{3} P_{2,1,0}^{o}$ threshold region. In general, one should be careful in interpreting resonances close to an ionization limit because spurious excursions may arise

TABLE V. Level energies $(E)$, ionization energies, and effective quantum numbers $\left(n^{*}\right)$ of the $\mathrm{Cl} 3 s 3 p^{5}\left({ }^{1} P_{1}^{o}\right) n p$ autoionizing Rydberg series.

\begin{tabular}{clcccc}
\hline & \multicolumn{2}{c}{${ }^{1} P_{1}^{o}$ series $^{\mathrm{a}}$} & & \multicolumn{2}{c}{${ }^{1} P^{o}$ series $^{\mathrm{b}}$} \\
\cline { 2 - 3 }$n$ & $E(\mathrm{eV})$ & $n^{*}$ & & $E(\mathrm{eV})$ & $n^{*}$ \\
\hline 4 & 24.631 & 2.25 & 24.84 & 2.33 \\
$5^{\mathrm{c}}$ & & & 26.14 & 3.34 \\
6 & 26.596 & 4.34 & 26.64 & 4.35 \\
7 & 26.834 & 5.31 & & \\
8 & 26.976 & 6.32 & & \\
9 & 27.065 & 7.35 & & \\
10 & 27.121 & 8.33 & & \\
$\infty^{\mathrm{d}}$ & \multicolumn{5}{c}{27.307} \\
$\infty^{\mathrm{c}}$ & \multicolumn{2}{c}{33.203} & & \\
$\infty^{\mathrm{f}}$ & & & & \\
\hline \hline
\end{tabular}

${ }^{\text {a }}$ This work; the estimated uncertainty in the experimental level energies is $\pm 10 \mathrm{meV}$.

${ }^{\text {b}}$ Theoretical results determined from Fig. 14 of Brown, Carter, and Kelly [12]; the experimental ionization energies used in this calculation and in the determination of $n^{*}$ are from Ref. [27].

${ }^{\mathrm{c}}$ The $n=5$ resonance could not be detected experimentally.

${ }^{\mathrm{d}}$ Ionization energy obtained by fitting the level energies to the Rydberg formula.

${ }^{e}$ Optical spectroscopy values based on the first ionization energy of $\mathrm{Cl}$ I [30] and the $\mathrm{Cl}$ II $3 s \rightarrow 3 p$ excitation energies of Moore [27]; see text.

fOptical spectroscopy values based on the first ionization energies of $\mathrm{Cl}$ I [30] and the $\mathrm{Cl}$ II $3 s \rightarrow 3 p$ excitation energies of Radziemski and Kaufman [33]; see text. from the finite resolution of the monochromator [74]. However, a comparison between the line shapes of the $4 p$ and $6 p$ resonances shows that the structure around 24.63 $\mathrm{eV}$ can be unambiguously identified as the $n=4$ resonance.

The level energies, the ionization energy, and the effective quantum numbers of the $3 s 3 p^{5}\left({ }^{1} P_{1}^{o}\right) n p$ Rydberg series are listed in Table $V$. The ionization energy and the effective quantum numbers were obtained by fitting the level energies $(n \geq 6)$ to the Rydberg formula [63]. The quantum defect $\delta$ was found to be about 1.65 , very close to the values obtained for the $\mathrm{Cl} 3 s 3 p^{5}\left({ }^{3} \mathrm{P}_{2,1,0}^{o}\right) n p$ and the Ar $3 s 3 p^{6}\left({ }^{2} S_{1 / 2}^{e}\right) n p$ Rydberg series.

Also included in Table $\mathrm{V}$ are the theoretical level energies of the first few members of the $\left({ }^{1} P_{1}^{o}\right) n p$ Rydberg series calculated by Brown, Carter, and Kelly [12]. As for the ${ }^{3} P_{2,1,0}^{o}$ resonances, these calculated values, which we obtained from Fig. 14 of Ref. [12], may have to be shifted downwards by $50 \mathrm{meV}$. The agreement between the experimental and the theoretical level energies of the $6 p$ resonances is quite good. Using our experimental values for the ${ }^{1} P_{1}^{o}$ limit and the quantum defect of the $\left({ }^{1} P_{1}^{o}\right) n p \quad$ Rydberg series (based on $n \geq 6$ ), the $3 s 3 p^{5}\left({ }^{1} P_{1}^{o}\right) 4 p$ and $3 s 3 p^{5}\left({ }^{1} P_{1}^{o}\right) 5 p$ resonance energies are calculated to be 24.836 and $26.098 \mathrm{eV}$, respectively, again in near-perfect agreement with theory. The actual position of the $n=4$ resonance is shifted downwards by approximately $0.20 \mathrm{eV}$ to $24.631 \mathrm{eV}$, very close to the ${ }^{3} P_{1}^{o}$ threshold. The MBPT calculation [12] may have slightly misjudged the interactions between the high- $n\left({ }^{3} P_{2,1,0}^{o}\right) n p$ Rydberg levels, on the one hand, and the $\left({ }^{1} P_{1}^{o}\right) 4 p$ levels, on the other. Experimentally, the $n=5$ resonance could not be positively identified because of the perturbing influence of nearby $3 s^{2} 3 p^{3} n n^{\prime} l^{\prime}$ states. These doubly excited states were not considered in the calculation of Brown, Carter, and Kelly [12].

As can be seen in Table $\mathrm{V}$, very good agreement between our experimental ${ }^{1} P_{1}^{o}$ ionization energy and the optical spectroscopy value based on the $\mathrm{Cl}$ II spectrum is obtained provided the $\mathrm{Cl}$ II assignments of Moore [27] are used. While there is little doubt about the positions of the $\mathrm{Cl}$ II $3 s 3 p^{5}\left({ }^{3} P_{2,1,0}^{o}\right)$ levels, which have been located about $11.65 \mathrm{eV}$ above the $\mathrm{Cl}$ II ${ }^{3} P_{2}^{e}$ ground state by both Moore [27] and Radziemski and Kaufman [33], a serious discrepancy arose with regard to the location of the $\mathrm{Cl}$ II $3 s 3 p^{5}\left({ }^{1} P_{1}^{o}\right)$ state. Moore [27] assigned the 14.34-eV level in $\mathrm{Cl} I \mathrm{I}$ to the $3 s 3 p^{5}\left({ }^{1} \mathrm{P}_{1}^{o}\right)$ state. Alternatively, on the basis of a $\mathrm{Cl}$ calculation, Radziemski and Kaufman [33] identified the $14.34-\mathrm{eV}$ level as the $3 s^{2} 3 p^{3}\left({ }^{2} D^{o}\right) 3 d\left({ }^{1} P_{1}^{o}\right)$ ionic state and ascribed the $\mathrm{Cl}$ II $20.24-\mathrm{eV}$ level to the $3 s 3 p^{5}\left({ }^{1} P_{1}^{o}\right)$ configuration. Clearly, the assignments of Radziemski and Kaufman [33] are not supported by the present experiments.

The $3 s^{2} 3 p^{3} n l n^{\prime} l^{\prime}$ level energies are listed in Table VI. From Fig. 10 it is clear that the intensity of the resonances corresponding to these doubly excited states is comparable to those related to the singly excited $3 s 3 p^{5}\left({ }^{1} P_{1}^{o}\right) n p$ states. The profiles of the $3 s^{2} 3 p^{3} n l n^{\prime} l^{\prime}$ resonances are seen to depend strongly on the continuum channel. A similar behavior was observed in the case of 
TABLE VI. Resonance energies (this work; the estimated uncertainty in the level energies is $\pm 10 \mathrm{meV}$ ) of the doubly excited $3 s^{2} 3 p^{3} n \ln ^{\prime} l^{\prime}$ resonances in atomic chlorine between 24.25 and $27.75 \mathrm{eV}$. The numbers refer to the resonances indicated in Figs. 10 and 11.

\begin{tabular}{cccc}
\hline Number & Energy $(\mathrm{eV})$ & Number & Energy $(\mathrm{eV})$ \\
\hline 1 & 25.054 & 10 & 26.289 \\
2 & 25.225 & 11 & 27.184 \\
3 & 25.391 & 12 & 27.219 \\
4 & 25.694 & 13 & 27.249 \\
5 & 25.769 & 14 & 27.299 \\
6 & 26.019 & 15 & 27.334 \\
7 & 26.054 & 16 & 27.434 \\
8 & 26.114 & 17 & 27.464 \\
9 & 26.174 & 18 & 27.619 \\
\hline \hline
\end{tabular}

$\mathrm{Br}$ [37], $\mathrm{Kr}$ [44-47], and $\mathrm{Xe}$ [44-47].

The $3 s^{2} 3 p^{3} n l n^{\prime} l^{\prime}$ configuration have three unfilled shells leading to many dipole-allowed electronic states and a potentially very rich spectrum. However, as in $\mathrm{Ar}$ [43], the $3 s^{2} 3 p^{3} 3 d n^{\prime} p, n^{\prime} f$ and $3 s^{2} 3 p^{3} 4 s n^{\prime} p, n^{\prime} f$ configurations are, as far as the doubly excited states are concerned, expected to dominate the spectrum. Note that because of parity conservation, $l l^{\prime}$ is restricted to be either $s p, s f, p d$, or $d f$. Unfortunately, the spectra in Fig. 10 do not provide clear evidence for additional Rydberg series converging upon any of the electronic states belonging to the $\mathrm{Cl}$ II $3 s^{2} 3 p^{3} 3 d$ and $3 s^{2} 3 p^{3} 4 s$ configurations. Hence, none of the resonances included in Table VI has been assigned to a particular electronic state. Several $3 s^{2} 3 p^{3} n l n^{\prime} l^{\prime}$ resonances lie above the $3 s^{-1}{ }^{1} P_{1}^{o}$ ionization limit at $27.316 \mathrm{eV}$, and it appears likely that the region between $27.75 \mathrm{eV}$ and the double ionization threshold of atomic chlorine at $36.77 \mathrm{eV}$ [27] will contain many more. A detailed study of this part of the $\mathrm{Cl}$ spectrum may help in identifying the resonances in the present experiments.

The absolute total $3 p^{-1}$ photoionization cross section of atomic chlorine across the $3 s 3 p^{5}\left({ }^{1} P_{1}^{o}\right) n p$ resonance region is shown in Fig. 11. The total $3 p^{-1}$ cross section has been obtained by adding the partial cross sections of Fig. 10. Because of the different line shapes, the resonances add up destructively and the intensity of many resonances is significantly reduced. With regard to the $3 s 3 p^{5}\left({ }^{1} P_{1}^{o}\right) n p$ Rydberg series, only the small $n=4$ and 6 windows can still be detected.

The total $\left(3 p^{-1}+3 s^{-1}\right)$ photoionization cross section in this region has been calculated by Brown, Carter, and Kelly [12]. In contrast to the rather weak features in our total $3 p^{-1}$ cross section, they obtained fairly intense, dispersion-shape autoionization profiles. This discrepancy may of course be due to the $3 s 3 p^{5}\left({ }^{3} P_{2,1,0}^{o}\right) \varepsilon p$ channel included in the MBPT calculation [12], but omitted from the total $3 p^{-1}$ cross section displayed in Fig. 11. Unfortunately, we have been unable to study the $3 s^{-1}{ }^{3} P_{2,1,0}^{o}$ partial cross sections across the $3 s 3 p^{5}\left({ }^{1} P_{1}^{o}\right) n p$ autoionizing Rydberg series because of experimental problems associated with accurately detecting the low-kinetic-energy photoelectrons. However, it seems unlikely that the

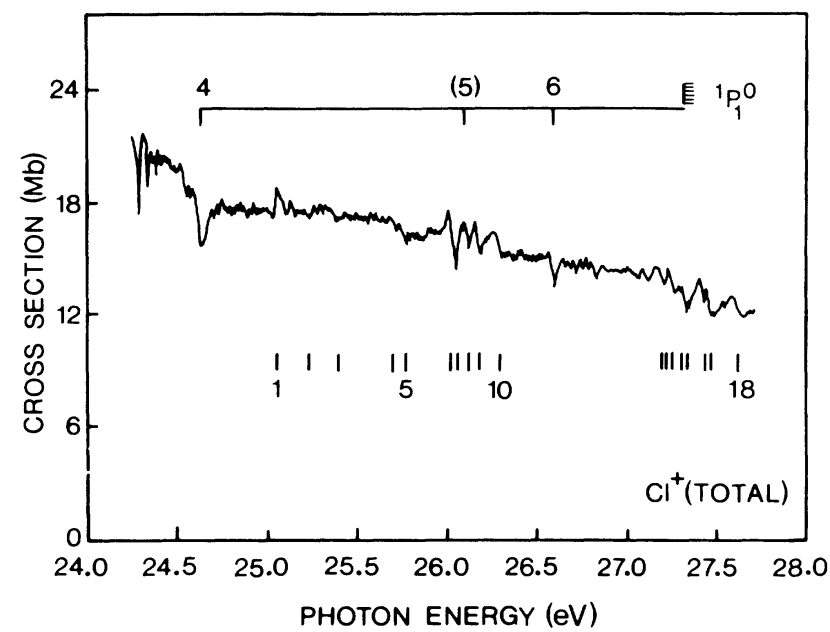

FIG. 11. Absolute total $3 p^{-1}$ photoionization cross section of atomic chlorine across the $3 s 3 p^{5}\left({ }^{1} P_{1}^{o}\right) n p(n \geq 4)$ resonance region between 24.25 and $27.75 \mathrm{eV}$. The total $3 p^{-1}$ cross section has been obtained by adding the partial cross sections of Fig. 10.

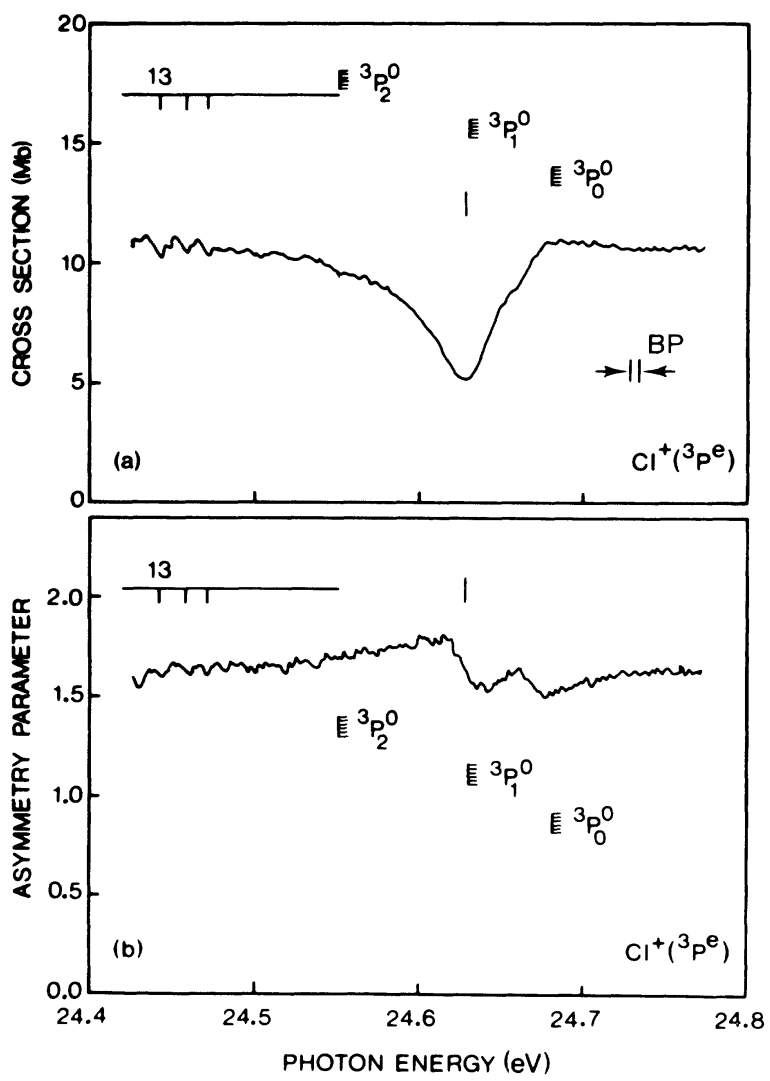

FIG. 12. (a) Absolute partial cross section and (b) asymmetry parameter of the $\mathrm{Cl}^{+}{ }^{3} P^{3}$ ionic state across the $3 s 3 p^{5}\left({ }^{1} P_{1}^{o}\right) 4 p$ autoionizing resonances between 24.45 and $24.80 \mathrm{eV}$. The positions of the highest members of the $3 s 3 p^{5}\left({ }^{3} P_{2}^{o}\right) n p$ Rydberg series and the locations of the $3 s^{-1}{ }^{3} P_{2}^{o},{ }^{3} P_{1}^{o}$, and ${ }^{3} P_{0}^{o}$ ionization thresholds are indicated. BP represents the monochromator bandpass of $0.11 \AA$ (FWHM) at which these spectra were recorded. The vertical tickmark corresponds to the minimum of the $4 p$ resonances in the ${ }^{3} P^{e}$ channel at $24.631 \mathrm{eV}$. These CIS spectra contain 350 channels each. 
small $3 s 3 p^{5}\left({ }^{3} P_{2,1,0}^{o}\right) \varepsilon p$ channel, whose nonresonant background cross section is of the order of $1.5 \mathrm{Mb}[11,12]$, could fully account for the difference between theoretical and experimental profiles. Thus, while the intensity of the $3 s 3 p^{5}\left({ }^{3} P_{2,1,0}^{o}\right) n p$ resonances has been underestimated by theory, the intensity of the $3 s 3 p^{5}\left({ }^{1} P_{1}^{o}\right) n p$ resonances appears to have been overestimated.

The absolute photoionization cross sections and the asymmetry parameters of the $3 p^{-13} P^{e},{ }^{1} D_{2}^{e}$, and ${ }^{1} S_{0}^{e}$ ionic states across the $3 s 3 p^{5}\left({ }^{1} P_{1}^{o}\right) 4 p$ resonances are shown in Figs. 12-14. The CIS spectra have been recorded at a resolution of $0.11 \AA$ (FWHM), while a stepsize of $1 \mathrm{meV}$ was used. The positions of the highest members of the $\left({ }^{3} P_{2}^{o}\right) n p$ Rydberg series, the locations of the ${ }^{3} P_{2}^{o},{ }^{3} P_{1}^{o}$, and ${ }^{3} P_{0}^{o}$ ionization thresholds, and a mark representing the bandpass of the monochromator are included in the figures. The vertical tickmark corresponds to the minimum of the $n=4$ resonance in the ${ }^{3} P^{e}$ channel. For completeness, the total $3 p^{-1}$ photoionization cross section of atomic chlorine in the same energy region is portrayed in Fig. 15.
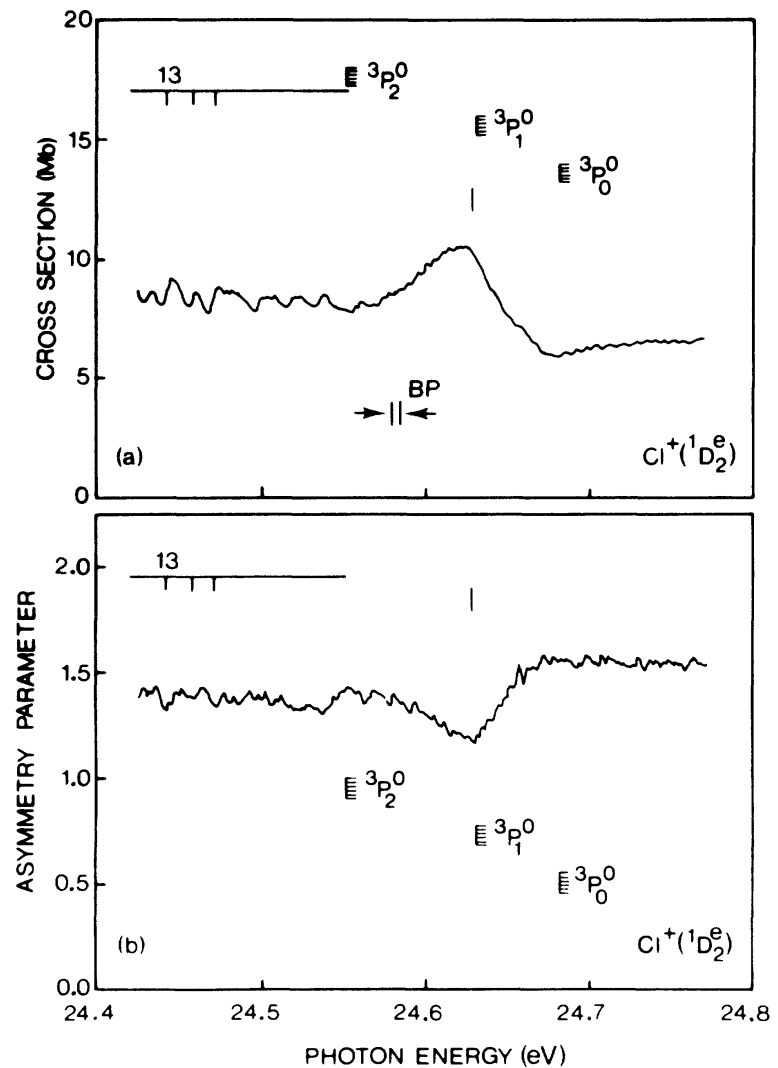

FIG. 13. (a) Absolute partial cross section and (b) asymmetry parameter of the $\mathrm{Cl}^{+}{ }^{1} D_{2}^{e}$ ionic state across the $3 s 3 p^{5}\left({ }^{1} P_{1}^{o}\right) 4 p$ autoionizing resonances between 24.45 and $24.80 \mathrm{eV}$. The positions of the highest members of the $3 s 3 p^{5}\left({ }^{3} P_{2}^{o}\right) n p$ Rydberg series and the locations of the $3 s^{-1}{ }^{3} P_{2}^{o},{ }^{3} P_{1}^{o}$, and ${ }^{3} P_{0}^{o}$ ionization thresholds are indicated. BP represents the monochromator bandpass of $0.11 \AA$ (FWHM) at which these spectra were recorded. The vertical tickmark corresponds to the minimum of the $4 p$ resonances in the ${ }^{3} P^{e}$ channel at $24.631 \mathrm{eV}$. These CIS spectra contain 350 channels each.
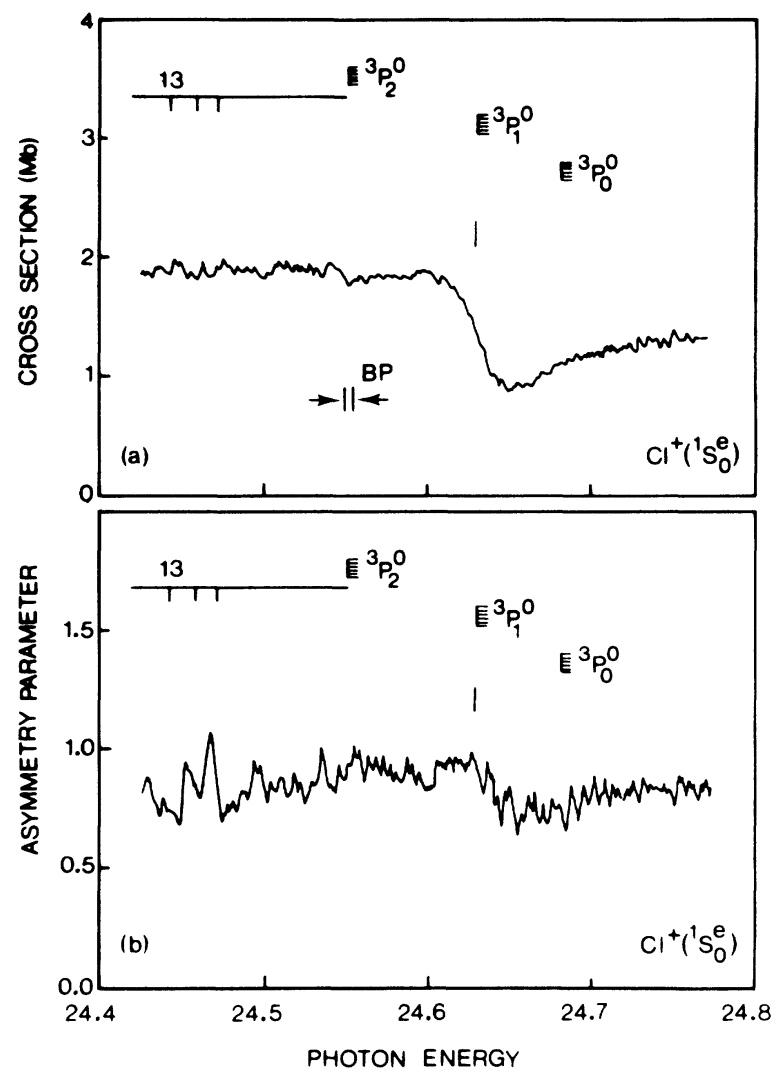

FIG. 14. (a) Absolute partial cross section and (b) asymmetry parameter of the $\mathrm{Cl}^{+}{ }^{1} S_{0}^{e}$ ionic state across the $3 s 3 p^{5}\left({ }^{1} P_{1}^{o}\right) 4 p$ autoionizing resonances between 24.45 and $24.80 \mathrm{eV}$. The positions of the highest members of the $3 s 3 p^{5}\left({ }^{3} P_{2}^{o}\right) n p$ Rydberg series and the locations of the $3 s^{-1},{ }^{3} P_{2}^{o},{ }^{3} P_{1}^{o}$, and ${ }^{3} P_{0}^{o}$ ionization thresholds are indicated. BP represents the monochromator bandpass of $0.11 \AA$ (FWHM) at which these spectra were recorded. The vertical tickmark corresponds to the minimum of the $4 p$ resonances in the ${ }^{3} P^{e}$ channel at $24.631 \mathrm{eV}$. These CIS spectra contain 350 channels each.

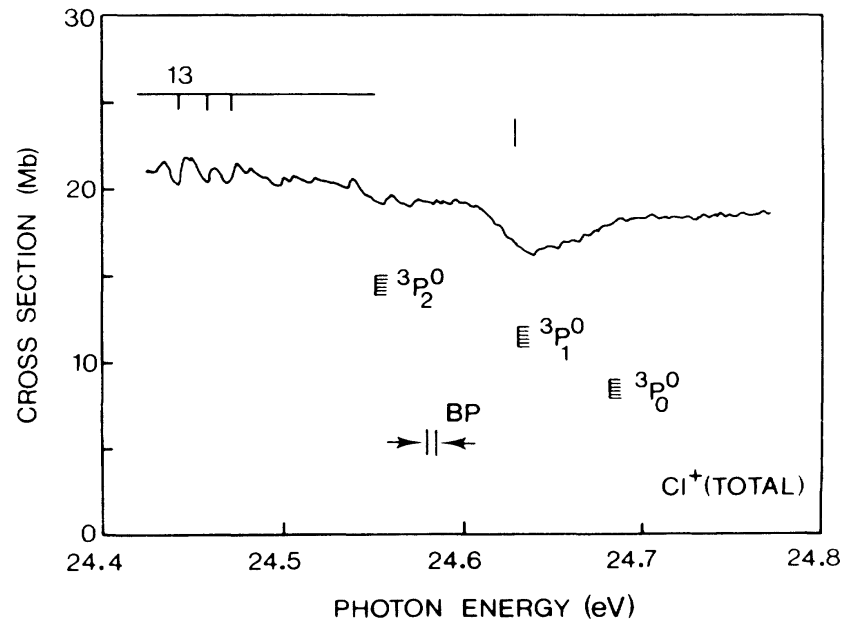

FIG. 15. Absolute total $3 p^{-1}$ photoionization cross section of atomic chlorine across the $3 s 3 p^{5}\left({ }^{1} P_{1}^{o}\right) 4 p$ autoionizing resonances between 24.45 and $24.80 \mathrm{eV}$. The total cross section has been obtained by adding the partial cross sections of Figs. $12-14$. 
Figures 12(a)-14(a) clearly illustrate the different cross-section profiles in the various $3 p^{-1}$ channels. A thorough understanding of these variations will require the use of elaborate quantum-mechanical calculations. The resonances in the asymmetry parameters are quite small. The underlying structure of the $3 s 3 p^{5}\left({ }^{1} P_{1}^{o}\right) 4 p$ manifold, which contains five nearly overlapping levels, may be partly resolved in Fig. 12(b), but the other spectra do not show any evidence of the splitting between the $4 p$ Rydberg levels. All $3 s 3 p^{5}\left({ }^{1} P_{1}^{o}\right) 4 p$ features observed are quite broad, and their width is much larger than the monochromator bandpass of about $5.4 \mathrm{meV}$. This large width may be the result of an effective decay pathway offered to the $3 s 3 p^{5}\left({ }^{1} P_{1}^{o}\right) 4 p$ Rydberg levels by the $3 s^{-1}$ $3 s 3 p^{5}\left({ }^{3} P_{2}^{o}\right) \varepsilon p$ channel which just opened up.

To corroborate our analysis of the $3 s 3 p^{5}\left({ }^{3} P_{2,1,0}^{o}\right) n p$ and $3 s 3 p^{5}\left({ }^{1} P_{1}^{o}\right) n p$ Rydberg series, we have recorded the $\mathrm{Cl} 3 s^{-1}$ photoelectron spectrum displayed in Fig. 16. This spectrum has been measured at a photon energy of $29.00 \mathrm{eV}$ and at an angle of $0^{\circ}$ with respect to the main polarization vector of the synchrotron radiation. Signals due to molecular chlorine have already been subtracted and the remaining background is presumably caused by low-kinetic-energy electrons generated inside the microwave discharge. The PE spectrum has been calibrated using the known ionization energies of $\mathrm{Ne} 2 p$ [38] and $\mathrm{Ar}$ $3 s$ [43], using $h v=31.00 \mathrm{eV}$ in the latter case.

The $\mathrm{Cl} 3 s^{-1}{ }^{3} P_{2,1,0}^{o}$ and ${ }^{1} P_{1}^{o}$ peaks are easily detected. The PE resolution is insufficient to resolve the ${ }^{3} P_{2}^{o},{ }^{3} P_{1}^{o}$, and ${ }^{3} P_{0}^{o}$ states. Using the top of the ${ }^{3} P_{2,1,0}^{o}$ and ${ }^{1} P_{1}^{o}$ peaks, the binding energies obtained are $24.549 \pm 0.015 \mathrm{eV}$ and $27.306 \pm 0.025 \mathrm{eV}$, respectively, in good agreement with

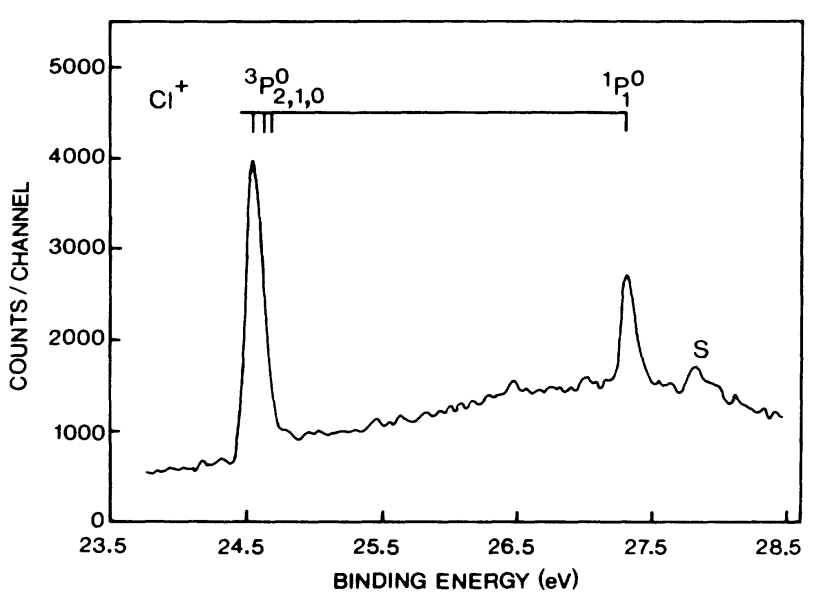

FIG. 16. $\mathrm{Cl} 3 s^{-1}$ photoelectron spectrum recorded at a photon energy of $29.00 \mathrm{eV}$ and at an angle of $0^{\circ}$ with respect to the main polarization vector of the synchrotron radiation. Signals due to molecular chlorine have been subtracted. The $3 s^{-1}$ ${ }^{3} P_{2,1,0}^{o}$ and ${ }^{1} P_{1}^{o}$ peaks are easily identified. The ionization energies determined from the Rydberg series are indicated in the figure, and good agreement with the independently calibrated PE spectrum is obtained. The small peak labeled $\mathrm{S}$ at $27.81 \mathrm{eV}$ has been assigned to the $3 s^{2} 3 p^{3}\left({ }^{4} S^{o}\right) 3 d\left({ }^{2} D_{3,2,1}^{o}\right)$ correlation satellite. The spectrum contains 256 channels. the values determined from the Rydberg series. The intensity ratio between the ${ }^{3} P_{2,1,0}^{o}$ and ${ }^{1} P_{1}^{o}$ signals, as derived from $\theta=0^{\circ} \mathrm{PE}$ spectra and uncorrected for angular distribution effects, is about $3.8 \pm 0.8$. The large error bar reflects the uncertainties in the analyzer response for low-kinetic-energy electrons and possible errors introduced in subtracting the background. Nevertheless, the intensity ratio appears to be larger than the geometric value of 3.0. On the basis of the $\mathrm{Cl}$ II energies listed by Moore [27], the small peak at $27.81 \mathrm{eV}$ has been tentatively assigned to the

$$
3 s^{2} 3 p^{3}\left({ }^{4} S^{o}\right) 3 d\left({ }^{2} D_{3,2,1}^{o}\right)
$$

correlation satellite.

\section{CONCLUSIONS}

Using synchrotron-radiation-based angle-resolved photoelectron spectrometry, we have determined the relative partial photoionization cross sections and asymmetry parameters of the $\mathrm{Cl} 3 p^{-1}{ }^{3} P_{2,1,0}^{e},{ }^{1} D_{2}^{e}$, and ${ }^{1} S_{0}^{e}$ ionic states across the $3 s 3 p^{5}\left({ }^{3} P_{2,1,0}^{o}\right) n p$ and $3 s 3 p^{5}\left({ }^{1} P_{1}^{o}\right) n p$ resonance regions between 21.75 and $27.75 \mathrm{eV}$. In addition, we measured the partial cross sections and the asymmetry parameters at the $\mathrm{He}$ I $\alpha$ energy $(21.218 \mathrm{eV})$. By combining the present results with the absolute $\operatorname{HeI} \alpha{ }^{3} P_{2,1,0}^{e}$ cross section obtained previously in an electron spectroscopy modulation experiment, we have been able to place all partial cross sections on an absolute scale. The best overall description of our measurements at $21.218 \mathrm{eV}$ is offered by those calculations which employ an open-shell transition-matrix approach.

Exploiting the high resolution of the 4-m NIM, the $3 s 3 p^{5}\left({ }^{3} P_{2,1,0}^{o}\right) n p$ and the $3 s 3 p^{5}\left({ }^{1} P_{1}^{o}\right) n p$ Rydberg series could be studied in detail. The $\left({ }^{3} P_{2}^{o}\right) n p,\left({ }^{3} P_{1}^{o}\right) n p$, and $\left({ }^{3} P_{0}^{o}\right) n p$ Rydberg series were clearly resolved and levels up to $n=14$ have been identified. The $\left({ }^{1} P_{1}^{o}\right) n p$ series could be observed up to $n=10$. The ${ }^{3} P_{2,1,0}^{o}$ and ${ }^{1} P_{1}^{o}$ ionization thresholds obtained from the Rydberg series agree very well with the ionization limits calculated on the basis of the $\mathrm{Cl}$ II spectrum, and with the ionization energies determined from a $3 s^{-1}$ photoelectron spectrum.

The quantum defects of the $\left({ }^{3} P_{2,1,0}^{o}\right) n p$ and the $\left({ }^{1} P_{1}^{o}\right) n p$ Rydberg series closely resembled those of the $\mathrm{Ar}$ $3 s 3 p^{6}\left({ }^{2} S_{1 / 2}^{e}\right) n p$ series. Furthermore, the integrated excess cross sections across the $\mathrm{Cl} 3 s 3 p^{5}\left({ }^{3} P_{2,1,0}^{o}\right) 5 p$ and the $\operatorname{Ar} 3 s 3 p^{6}\left({ }^{2} S_{1 / 2}^{3}\right) 5 p$ manifolds were found to be virtually identical. However, the detailed $\mathrm{Cl}$ autoionization profiles were much more complex, reflecting the openshell character of the chlorine atom. The $\left({ }^{3} P_{2,1,0}^{o},{ }^{1} P_{1}^{o}\right) n p$ level energies are well reproduced by a nonrelativistic MBPT calculation. The agreement between experimental and theoretical line shapes is less satisfactory, in particular for the $\left({ }^{1} P_{1}^{o}\right) n p$ resonances.

Resonances due to the $3 s^{2} 3 p^{5} \rightarrow 3 s^{2} 3 p^{3} n \ln ^{\prime} l^{\prime}$ twoelectron excitations have been observed in the ${ }^{1} P_{1}^{o}$ resonance region. These doubly excited states may be responsible for the missing $5 p$ member of the $3 s 3 p^{5}\left({ }^{1} P_{1}^{o}\right) n p$ Rydberg series. The line shapes of the two-electron resonances were seen to depend strongly on the continuum 
channel. None of the $3 s^{2} 3 p^{3} n l n^{\prime} l^{\prime}$ resonances has been assigned.

In order to help characterize the as-yet-unidentified two-electron resonances, an extension of this work into the energy region between $27.75 \mathrm{eV}$ and the $\mathrm{Cl}$ double ionization threshold at $36.77 \mathrm{eV}$ would seem worthwhile. In addition, our understanding of the role of electron correlations in the photoionization of atomic chlorine will certainly benefit from a comparison between the present experimental results and new, detailed quantummechanical calculations incorporating relativistic effects and considering the behavior of the partial cross sections and asymmetry parameters across the resonances.

\section{ACKNOWLEDGMENTS}

This work has been supported by the U.S. Department of Energy, under Contract No. DE-AC05-84OR21400 with the Martin Marietta Energy Systems, Inc. Financial support from the Netherlands Organization for Scientific Research (PvdM) and the North Atlantic Treaty Organization under Grant No. 0404/87 is gratefully acknowledged. C.D.C. and S.B.W. were sponsored by the U.S. National Science Foundation under Grant No. PHY8907286. The Synchrotron Radiation Center is operated under U.S. National Science Foundation Grant No. DMR-8821625.
[1] J. A. R. Samson, in Corpuscles and Radiation in Matter, edited by W. Mehlhorn, Hanbuch der Physik Vol. 31 (Springer-Verlag, Berlin, 1982) p. 123; A. F. Starace, ibid., p. 1.

[2] A. F. Starace, R. H. Rast, and S. T. Manson, Phys. Rev. Lett. 38, 1522 (1977).

[3] S. T. Manson, A. Msezane, A. F. Starace, and S. Shahabi, Phys. Rev. A 20, 1005 (1979).

[4] S. T. Manson and A. F. Starace, Rev. Mod. Phys. 54, 389 (1982).

[5] M. J. Conneely, K. Smith, and L. Lipsky, J. Phys. B 3, 493 (1970).

[6] A. F. Starace and L. Armstrong, Phys. Rev. A 13, 1850 (1976).

[7] N. A. Cherepkov and L. V. Chernysheva, Phys. Lett. A 60, 103 (1977).

[8] N. A. Cherepkov and L. V. Chernysheva, Izv. Akad. Nauk SSSR, Ser. Fiz. 41, 2518 (1977) [Bull. Acad. Sci. USSR, Phys. Ser. 41, 47 (1977)].

[9] V. G. Yarzhemsky and V. I. Nefedov, J. Electron Spectrosc. Relat. Phenom. 23, 175 (1981).

[10] M. Lamoureux and F. Combet Farnoux, J. Phys. (Paris) 40, 545 (1979).

[11] E. R. Brown, S. L. Carter, and H. P. Kelly, Phys. Lett. A 66, 290 (1978).

[12] E. R. Brown, S. L. Carter, and H. P. Kelly, Phys. Rev. A 21, 1237 (1980).

[13] W. R. Fielder and L. Armstrong, Phys. Rev. A 28, 218 (1983).

[14] Z.-W. Wang and K. T. Lu, Phys. Rev. A 31, 1515 (1985).

[15] Z.-W. Wang and K. T. Lu, Phys. Rev. A 31, 1521 (1985).

[16] S. Shahabi, A. F. Starace, and T. N. Chang, Phys. Rev. A 30, 1819 (1984).

[17] S. Shahabi and A. F. Starace, Phys. Rev. A 33, 2111 (1986).

[18] J. E. Hansen, R. D. Cowan, S. L. Carter, and H. P. Kelly, Phys. Rev. A 30, 1540 (1984).

[19] Z.-D. Qian, S. L. Carter, and H. P. Kelly, Phys. Rev. A 33, 1751 (1986).

[20] D. M. de Leeuw, R. Mooyman, and C. A. de Lange, Chem. Phys. Lett. 54, 231 (1978).

[21] K. Kimura, T. Yamazaki, and Y. Achiba, Chem. Phys. Lett. 58, 104 (1978).

[22] W. J. van der Meer, R. Butselaar, and C. A. de Lange, Aust. J. Phys. 39, 779 (1986).

[23] J. B. Peel and E. Von Nagy-Felsobuki, Spectrochim. Acta B 43, 217 (1988).
[24] P. van der Meulen, M. O. Krause, C. D. Caldwell, S. B. Whitfield, and C. A. de Lange, J. Phys. B 24, L573 (1991).

[25] B. Ruscic and J. Berkowitz, Phys. Rev. Lett. 50, 675 (1983).

[26] J. A. R. Samson, Y. Shefer, and G. C. Angel, Phys. Rev. Lett. 56, 2020 (1986).

[27] C. E. Moore, in Atomic Energy Levels, Natl. Bur. Stand. (U.S.) Circ. No. 467 (U.S. GPO, Washington, DC 1949).

[28] R. E. Huffman, J. C. Larrabee, and Y. Tanaka, J. Chem. Phys. 47, 856 (1967).

[29] R. E. Huffman, J. C. Larrabee, and Y. Tanaka, J. Chem. Phys. 48, 3835 (1968).

[30] L. J. Radziemski and V. Kaufman, J. Opt. Soc. Am. 59, 424 (1969).

[31] A. M. Cantu, W. H. Parkinson, T. Grisendi, and G. Tagliaferri, Phys. Scr. 31, 578 (1985).

[32] A. M. Cantu and W. H. Parkinson, Phys. Scr. 37, 336 (1988).

[33] L. J. Radziemski and V. Kaufman, J. Opt. Soc. Am. 64, 366 (1974).

[34] M. O. Krause, T. A. Carlson, and A. Fahlman, Phys. Rev. A 30, 1316 (1984).

[35] P. van der Meulen, C. A. de Lange, M. O. Krause, and D. C. Mancini, Phys. Scr. 41, 837 (1990).

[36] P. van der Meulen, M. O. Krause, and C. A. de Lange, Phys. Rev. A 43, 5997 (1991).

[37] P. van der Meulen, M. O. Krause, and C. A. de Lange, J. Phys. B 25, 97 (1992).

[38] M. O. Krause, in Synchrotron Radiation Research, edited by H. Winick and S. Doniach (Plenum, New York, 1980), p. 101.

[39] G. V. Marr and J. B. West, At. Data Nucl. Data Tables 18, 497 (1976).

[40] S. H. Southworth, A. C. Parr, J. E. Hardis, J. L. Dehmer, and D. M. P. Holland, Nucl. Instrum. Methods A 246, 782 (1986).

[41] H. van Lonkhuyzen, Ph.D. thesis, Free University, Amsterdam, The Netherlands, 1984.

[42] M. O. Krause, F. Cerrina, and A. Fahlman, Phys. Rev. Lett. 50, 1118 (1983).

[43] R. P. Madden, D. L. Ederer, and K. Codling, Phys. Rev. 177, 136 (1969).

[44] K. Codling and R. P. Madden, Phys. Rev. A 4, 2261 (1971).

[45] K. Codling and R. P. Madden, J. Res. Natl. Bur. Stand. A 76, 1 (1972).

[46] D. L. Ederer, Phys. Rev. A 4, 2263 (1971). 
[47] D. L. Ederer, Phys. Rev. A 14, 1936 (1976).

[48] M. G. Flemming, J.-Z. Wu, C. D. Caldwell, and M. O. Krause, Phys. Rev. A 44, 1733 (1991).

[49] J. Kreile and A. Schweig, J. Electron Spectrosc. Relat. Phenom. 20, 191 (1980).

[50] V. Schmidt, Z. Phys. D 2, 275 (1986).

[51] F. Wuilleumier and M. O. Krause, J. Electron Spectrosc. Relat. Phenom. 15, 15 (1979).

[52] M. O. Krause, Phys. Fenn. 9, Suppl. S1, 281 (1974).

[53] K. Kimura, S. Katsumata, Y. Achiba, T. Yamazaki, and S. Iwata, Handbook of He I Photoelectron Spectra of Fundamental Organic Molecules (Halsted, New York, 1981), pp. 23, 29, and 31 .

[54] J. Berkowitz and G. L. Goodman, J. Chem. Phys. 71, 1754 (1979).

[55] S. Daviel, Y. Iida, F. Carnovale, and C. E. Brion, Chem. Phys. 83, 391 (1984).

[56] J. A. R. Samson and G. C. Angel, J. Chem. Phys. 86, 1814 (1987).

[57] W. J. van der Meer, P. van der Meulen, M. Volmer, and C. A. de Lange, Chem. Phys. 126, 385 (1988).

[58] J. A. R. Samson and P. N. Pareek, Phys. Rev. A 31, 1470 (1985).

[59] G. C. Angel and J. A. R. Samson, Phys. Rev. A 38, 5578
(1988).

[60] I. I. Sobelman, Atomic Spectra and Radiative Transitions (Springer-Verlag, Berlin, 1979).

[61] R. D. Cowan and K. L. Andrews, J. Opt. Soc. Am. 55, 502 (1965).

[62] S. T. Pratt, P. M. Dehmer, and J. L. Dehmer, Phys. Rev. A 43, 4702 (1991).

[63] M. J. Seaton, Rep. Prog. Phys. 46, 167 (1983).

[64] F. Combet Farnoux, Phys. Rev. A 25, 287 (1982).

[65] U. Fano, Phys. Rev. 124, 1866 (1961).

[66] U. Fano and J. W. Cooper, Phys. Rev. 137, A1364 (1965).

[67] U. Fano and J. W. Cooper, Rev. Mod. Phys. 40, 441 (1968).

[68] B. W. Shore, Rev. Mod. Phys. 39, 439 (1967).

[69] B. W. Shore, J. Opt. Soc. Am. 57, 881 (1967).

[70] B. W. Shore, Phys. Rev. 171, 43 (1968).

[71] F. H. Mies, Phys. Rev. 175, 164 (1968).

[72] W. A. Svensson, M. O. Krause, and T. A. Carlson, J. Phys. B 20, L271 (1987).

[73] P. van der Meulen, M. O. Krause, C. D. Caldwell, S. B. Whitfield, and C. A. de Lange (unpublished).

[74] J. Berkowitz, Photoabsorption, Photoionization, and Photoelectron Spectroscopy (Academic, New York, 1979). 